\title{
Eye movement trajectories in active visual search: Contributions of attention, memory, and scene boundaries to pattern formation
}

\author{
T. D. KEECH AND L. RESCA \\ Catholic University of America, Washington, D.C.
}

\begin{abstract}
We relate the roles of attention, memory, and spatial constraints to pattern formation in eye movement trajectories previously measured in a conjunctive visual search task. Autocorrelations and power spectra of saccade direction cosines confirm a bias to progress forwardly, while turning at the display boundaries, plus a long-range memory component for the search path. Analyses of certain measures of circulation and imbalance in the eye trajectories, and their relations with the display area correspondingly subtended, bear signatures of spiraling or circulating patterns. We interpret their prevalence as mainly due to the interactions between three basic psychoneural mechanisms (conspicuity area, forward bias, long-range memory) and two task-specific geometric-spatial constraints on the eye trajectories (central start and display confinement). Conversely, computer simulations of random walks in which all psychoneural mechanisms are eliminated, while geometric-spatial constraints are maintained, show no prevalence of circulating patterns by those measures. We did find certain peculiarities of some individual participants in their pattern selections, but they appear too casual and incidental to suggest more systematic or complex search strategies in our randomized displays of uninformative stimuli.
\end{abstract}

Recent years have witnessed a remarkable explosion of scientific interest in and understanding about major psychophysical processes and mechanisms that underlie visual search in natural scenes and in laboratory setups designed to investigate critical elements of such scenes and processes. Visual search generally proceeds through a series of eye movements and fixations that bring sequentially different areas of a scene under scrutiny by the eye fovea. Affecting the patterns of those eye movements and fixations is a complex interplay between the scene's structures and boundaries and the observer's intents and perceptual, attentional, and mnemonic resources. In the present study, we attempt to contribute some elements and techniques to the investigation of such pattern formation.

\section{Attentional Resources}

Effective use of attentional resources and deployment of visuospatial attention constitute principal elements in visual search. These have been widely studied and extensively characterized for quite some time, often resulting in different models and terminologies for describing essentially equivalent or related concepts and results. One such basic terminology and characterization of the attentional focus is that of the area of conspicuity (AC), which Engel $(1971,1974,1977)$ originally defined as the zone in which objects can be effectively and simultaneously processed for target recognition. Equivalent concepts and definitions have previously and subsequently appeared, such as those of functional visual field (FVF; Ikeda \& Takeuchi, 1975;
Sanders, 1970; Sanders \& Houtmans, 1985), useful field of view (Ball, Beard, Roenker, Miller, \& Griggs, 1988; Scialfa, Kline, \& Lyman, 1987; Scialfa, Thomas, \& Joffe, 1994), and visual lobe area (Kraiss \& Knäeuper, 1982; Widdel, 1983; Widdel \& Kaster, 1981).

During the last decade, definitive studies of the AC (or FVF) and its role in guiding eye movements and fixations have been conducted by Motter and his collaborators (Motter \& Belky, 1998a, 1998b; Motter \& Holsapple, 2000, 2001, 2007; Motter \& Simoni, 2007, 2008). For a conclusive review, we can do no better than to refer to the study of Motter and Simoni (2008) in particular. In the first paragraph of their introduction, they immediately point out that "the FVF boundary is not set by the acuity threshold of peripheral vision. Instead, the size of the FVF is dynamically controlled by two principal factors: (1) the discriminability between the targets and distracting objects that results from crowding interference between items [see quotations in original article] and (2) the attentive demands of the visual task in both the fovea and the periphery [see quotations in original article]" (p. 2382). In the next paragraph, they note that "during visual search through spatially homogenous stimulus arrays, target detection performance falls off as a function of increasing target eccentricity from the point of fixation. The probability of detecting a target as a function of eccentricity can be used to define the FVF boundary" (p. 2382). Deeper understanding of the FVF and its varying size and shape, reviewed in that article's discussion section, derives

L. Resca, resca@cua.edu 
from the connection between local crowding discrimination of distractors around a target in homogeneously and heterogeneously cluttered environments, on the one hand, and the corresponding separation of items in the mapping space of their cortical representation, on the other hand. Our experiments (Keech, 2006; Keech, Greenwood, Resca, \& Parasuraman, 2009) consisted of a visual search task and psychophysical measurements quite similar to those in Motter and Belky (1998a, 1998b). Detailed and extensive comparisons of our results with theirs revealed no essential discrepancy with regard to the characterization of the AC in all its features. We shall thus assume Motter's definition and characterization of the AC (or FVF) in this article as well.

Although the $\mathrm{AC}$ is at least clearly defined operationally, that does not exhaust the complexity of focal attention. For example, one can maintain foveal fixation on a given item or location while intentionally shifting focal attention to a different item or location. In that case, the spherical shape of the AC deforms and stretches approximately into an elliptical form, with one focus remaining at the eye fixation while the other focus shifts toward the attentional focus. Other functional and dynamical properties of the zone of focal attention have been described in terms of various spotlight metaphors and zoom-lens models (Castiello \& Umiltà, 1990; Cave \& Bichot, 1999; Downing, 1988; Egeth, 1977; Eriksen \& St. James, 1986; Greenwood \& Parasuraman, 1999, 2004; LaBerge \& Brown, 1989; N. G. Müller, Bartelt, Donner, Villringer, \& Brandt, 2003; Zelinsky, Rao, Hayhoe, \& Ballard, 1997). ${ }^{1}$ These conceptualizations are not inconsistent with the basic features of the AC (or FVF) characterization, but that is seldom emphasized. Instead, other features are highlighted, such as how the attentional focus may manage to shift, change, or turn on and off in space and time (Chastain, 1992; Eriksen \& Murphy, 1987; Murphy \& Eriksen, 1987; Saarinen \& Julesz, 1991; Sagi \& Julesz, 1985; Sperling \& Weichselgartner, 1995).

Although it is often possible to inspect a greater portion of a scene through intentional covert shifts of attention, while maintaining the eyes essentially stationary, humans naturally prefer to move their eyes. Making saccades while searching for a target among distractors, which we refer to as active visual search, indeed occurs spontaneously even when participants can locate the target faster if they do not make eye movements (Zelinsky \& Sheinberg, 1997). On the other hand, one may also wonder whether some other sort of unintentional covert shifts of attention could still occur sequentially during each eye fixation in an active visual search. That seems to contradict the basic principle of a spherical AC within which items are processed in parallel during active visual search, and it has indeed been shown, in a thorough study by Motter and Holsapple (2007), to be an unlikely mechanism.

What generates or influences eye movements more specifically is another question of much greater scope and complexity, to which some notable studies (Findlay, 1997; Findlay, Brown, \& Gilchrist, 2001; Findlay \& Walker, 1999; Hooge \& Erkelens, 1996, 1998, 1999; McPeek, Skavenski, \& Nakayama, 2000; Motter \& Belky, 1998a, 1998b; Najemnik \& Geisler, 2005; Unema, Pannasch, Joos, \& Velichkovsky, 2005; Zelinsky, 1996; Zelinsky \& Sheinberg, 1997; Zihl \& Hebel, 1997) and many others have contributed in various respects. In particular, a sequential model of attention has described how covert shifts of attention may produce overt movements of the eyes (Henderson, 1992, 1993), and it has been demonstrated that any saccade is immediately preceded by a covert shift of attention to the location where the eye fovea is prepared to land (Deubel \& Schneider, 1996; Kowler, Anderson, Dosher, \& Blaser, 1995; Peterson, Kramer, \& Irwin, 2004).

\section{Mnemonic Resources}

With regard to mnemonic resources, a remarkable quantity of recent studies of high quality have identified various memory types and components, operating separately, concurrently, or not at all, depending on the types and conditions of the visual search tasks. Various studies may be categorized, although not necessarily most pertinently, on the basis of the capacity of the memory that they suggest. Examples vary from little or no memory (Horowitz \& Wolfe, 1998, 2001, 2003; Woodman, Vogel, \& Luck, 2001) to limited memory capacity (Beck, Peterson, Boot, Vomela, \& Kramer, 2006; Boot, McCarley, Kramer, \& Peterson, 2004; Dodd, Castel, \& Pratt, 2003; Gibson, Li, Skow, Brown, \& Cooke, 2000; Gilchrist \& Harvey, 2000; Irwin \& Zelinsky, 2002; Klein \& MacInnes, 1999; McCarley, Wang, Kramer, Irwin, \& Peterson, 2003; Motter \& Holsapple, 2007; H. J. Müller \& von Mühlenen, 2000; Najemnik \& Geisler, 2005; Takeda \& Yagi, 2000; Thornton \& Horowitz, 2004; von Mühlenen, Müller, \& Müller, 2003; Wolfe, 2003; Woodman \& Luck, 2004; Zelinsky \& Loschky, 2005) to larger memory capacity (Aks, Zelinsky, \& Sprott, 2002; Dickinson \& Zelinsky, 2005, 2007; Gilchrist, North, \& Hood, 2001; Keech, 2006; Keech et al., 2009; Keech \& Resca, in press; Kristjánsson, 2000; Peterson, Kramer, Wang, Irwin, \& McCarley, 2001; Takeda, 2004). For succinct but more informative surveys of these and other, related studies, we can do no better than recommend and refer to the introductory sections of three articles in particular, which are also most relevant to our own studies - namely, those of Beck, Peterson, and Vomela (2006), Peterson, Beck, and Vomela (2007), and Dickinson and Zelinsky (2007).

Inhibition of return. No less critical than memory capacity is the identification of which memory components may be involved in visual search, depending on the task. Perhaps the most widely studied element is inhibition of return (IOR). It was actually discovered as an attentional mechanism in a cue-response paradigm (Posner \& Cohen, 1984; Posner, Rafal, Choate, \& Vaughan, 1985), but it was soon related to an inhibitory tagging system that facilitates visual search by preventing attention from returning to already inspected items (Klein, 1988). Klein and MacInnes (1999) demonstrated further that IOR acts as a foraging facilitator in visual search and, correspondingly, that IOR induces a directional forward bias in consecutive saccades - a feature that is also critical in our study. Although IOR may be extinguished under 
certain conditions (Gibson \& Egeth, 1994a; Klein \& Taylor, 1994; Wolfe \& Pokorny, 1990), it is typically robust, at least when search stimuli remain visible (H. J. Müller \& von Mühlenen, 2000; Takeda \& Yagi, 2000). In fact, IOR has been demonstrated for both object-based and environment-based locations (Gibson \& Egeth, 1994b), with presumably alternative supporting neural structures (Tipper, Weaver, Jerreat, \& Burak, 1994). Furthermore, it has been shown that the inhibitory tagging process can begin to accrue rather rapidly, making it possible for a memory-based IOR to facilitate visual search (Dodd et al., 2003). With regard to the memory capacity associated with IOR, at least three to five spatially noncontiguous locations have been confirmed, although with a declining magnitude of inhibition at the earlier sites (Danziger, Kingstone, \& Snyder, 1998; Snyder \& Kingstone, 2000). Over time, Klein and colleagues have provided excellent reviews of the rapidly growing literature on IOR, its neural correlates, and related issues (Klein, 2000; Lupiáñez, Klein, \& Bartolomeo, 2006; Shore \& Klein, 2000; Taylor \& Klein, 1998).

Search strategies. An alternative way of avoiding returns to previously fixated locations is to adopt search strategies that may be somewhat predetermined from the start and designed to scan sequentially and systematically the entire display until the target is located. This procedure may involve an even more automatic form of memory, since one needs to "remember" only how to follow (procedurally and unconsciously, typically) a preselected scanpath and where one is along that path at any given time. This becomes presumably a more economic and effective procedure for avoiding returns to previously fixated locations when the number of items requiring inspection grows substantially (beyond the capacity of IOR). On the other hand, such procedures may be either possible or beneficial only if stimulated or guided by the perception of certain regularities or patterns within the scene or at its boundaries (bottom-up processes) and if those are naturally compatible with physiological (Lévy-Schoen, 1981) and phylogenetic or idiosyncratic viewing biases characteristic of the subject (top-down processes).

Scanpaths in eye movements, both characteristic of each observing individual and peculiar to each observed scene, were originally characterized by Stark and colleagues in studies of perception and recognition of realistic but marginally visible drawings (Noton \& Stark, 1971; Stark \& Choi, 1996). Lately, Gilchrist and Harvey (2006) have demonstrated a systematic scanning, mainly left to right, which however was "almost certainly a function of the type of structure that was imposed on the displays: specifically, the display items were placed randomly on the intersections of a grid" (p. 712). So, although stimulating in many respects, these findings could have been expected, since the underlying gridlike structure of their displays is quite conspicuous and immediately perceived, even for the "least structured" displays with the fewest items (25) randomly placed on the junctions of an "imaginary," but still very noticeable, $7 \times 7$ grid (see their Figure 1). In retrospect, Gilchrist and Harvey (2006) could have performed some simple variations, such as rotating their gridlike displays by $45^{\circ}$, say, in order to determine whether the mainly horizontal systematic scanning would persist, disappear, or tilt. That could have been more revealing of possibly competing "innate," or "endogenous" (horizontal-vertical), and "guided," or "exogenous" (tilting), biases.

More elaborate studies of scanpath and saccade planning have been conducted by Findlay and Brown (2006a, 2006b), still suggesting various degrees of stereotypicality and systematicity, depending on the observers and the layout structure of multielement displays. Stereotypicality and top-down scanning strategies have also been demonstrated and quantified in relation to their ability to influence stimulus-driven attentional and oculomotor capture by abrupt onset stimuli (Peterson, Kramer, Irwin, \& Hahn, 2002). On the other hand, Scinto, Pillalamarri, and Karsh (1986) have concluded from experiments and computer simulations of visual search in texture structures that oculomotor behavior is more nearly random than directed by any systematic strategy in visually homogeneous fields with nonpreattentively discriminable targets. Manipulations of global field boundaries were also found to have little effect on performance in this type of search task, which is quite different from the previous ones, as well as from ours.

Peterson et al. (2007) have identified separate contributions of retrospective memory, comprising IOR, and prospective memory, which may result from scanning strategies planned ahead of the actual search performance. They compared participant performances between viewing stable displays and displays with changing conditions, in which either the items that had already been inspected or those that had not yet been inspected were relocated during each saccade (thus exploiting the phenomenon of saccadic suppression). As was expected, alternative display-changing conditions disrupted either retrospective or prospective memory, indicating a capacity of about four or five items for the retrospective component and a larger capacity for the prospective component. This may account for the apparent discrepancies among previous studies, which have alternatively suggested a limited memory capacity (e.g., Klein \& MacInnes, 1999; McCarley et al., 2003) and a larger memory capacity (e.g., Peterson et al., 2001). The stereotypicality of scanpaths was also investigated by Peterson et al. (2007), but that may have again been induced or affected by an underlying gridlike structure (see their Figures 4 and 6) similar to that in Gilchrist and Harvey (2006).

It may be hard to ascertain whether a scanpath is virtually planned in advance of the actual search or possibly reprogrammed or even made up as the visual search proceeds, since most of that occurs outside of awareness and beyond any conscious or intentional planning. Without committing to any planning, perhaps there is some coarse form of retrospective memory, quite separate from IOR, for whatever scanpath has been followed up to each point along the search, whether that scanpath was part of any systematic strategy or just happened to be realized for whatever reason. Although the experiments of Peterson et al. (2007) seem to exclude any such form of retrospective memory, those of Dickinson and Zelinsky (2007) seem to indicate 
a form of high-capacity (for at least 12 inspected items) but low-resolution memory for the search path. Rather than contradicting each other, these two well-conceived and well-conducted studies may confirm what some investigators (including ourselves) have felt all along - namely, that there may be "no one form of memory available for use during visual search, and the type of memory ultimately used may depend on the specific demands of the search task.... When we provided observers with a stable and accurate cue to the locations of the search objects, they were able to discriminate inspected from uninspected objects at points in their search histories that far exceeded traditional estimates of memory capacity during search" (Dickinson \& Zelinsky, 2007, p. 1754). Conversely, still paraphrasing Dickinson and Zelinsky (2007, p. 1754), display change paradigms used in studies so as to creatively test the limits of inhibition-based retrospective memory may have thwarted the use of a path memory. The results of our studies (Keech, 2006; Keech et al., 2009; Keech \& Resca, in press) are most consistent with these views, perhaps not surprisingly, since the items in our displays were both stably maintained and randomly distributed, just as in the displays used by Dickinson and Zelinsky (2007, Figure 1).

\section{Scene Boundaries}

Although it may not always be adequately recognized or appreciated, scene boundaries and constraints typically play critical roles in both conduct and type of many, if not most, visual searches. First of all, it serves no purpose to search for items outside of their natural or intended boundaries; hence, visual systems in many animal species should have evolved to become adept in perceiving or recognizing those boundaries and confining visual searches appropriately within them. Looking for fruits on a tree, fish in a pond, birds in a flock, prey in a herd, obstacles in the way, cars in the street, pedestrians on the sidewalk, players in a field, swimmers in a pool, pens on a desktop, clothes in a closet, books on the shelves, or items on a television screen or a computer monitor provide but a few common examples of how quickly and automatically people and animals can and must determine where to look and where not to look precisely. ${ }^{2}$ By contrast, when the scene has no natural or obvious boundaries, visual search can become much more problematic and unsettled, as a proverbial "predator" or "prey" may lurk or hide anywhere. Second, but also importantly, perceived structures and regularities at the scene boundaries may in turn induce or influence possible search strategies and scanpaths to be conducted within those confines.

In many, if not most, visual search studies, computer monitors and bounded displays have been used, but researchers often do not bother to investigate or report which fractions of, or under which conditions, eye movements may stray out of those bounds, for example. Such occurrences are presumed to be rare, as we found in our experiments. ${ }^{3}$ Most studies also do not specifically investigate the possible roles or influences that scene boundaries and constraints may have on the development of either scanning strategies or actual scanpaths. Vice versa, that investigation is a major component of our study.

\section{Visual Search Theories}

Well-known theories of visual search conduct include feature integration theory (FIT; Treisman, 1988; Treisman \& Gelade, 1980; Treisman \& Gormican, 1988; Treisman \& Sato, 1990), guided search theory (GST; Cave \& Wolfe, 1990; Wolfe, 1994, 1998; Wolfe, Cave, \& Franzel, 1989), attentional engagement theory (AET; Duncan \& Humphreys, 1989, 1992), signal detection theory (SDT; Eckstein, Thomas, Palmer, \& Shimozaki, 2000; Verghese, 2001), texton theory (Julesz, 1981, 1984; Julesz \& Bergen, 1983), and sequential attention theory (Henderson, 1992, 1993).

In a seminal investigation, in which participants were required to find targets in arrays of objects with relatively few distinct and simple features, Treisman and Gelade (1980) developed a corresponding FIT of attention. According to that theory, attention is directed serially to each stimulus in a display whenever conjunctions of more than one separable feature are needed to distinguish the stimuli. Although Treisman and Gelade originally measured only the reaction time needed to identify the target, Zelinsky and Sheinberg (1997) subsequently also tracked eye movements and showed that the number of saccades, in particular, is highly correlated with reaction time. Both measures are thus predictive of parallel-serial search dichotomies. That is, when target recognition requires only the detection of a single "pop-out" or primitive visual feature, such as either color or orientation, the visual search is performed in parallel, and there is essentially no dependence of number of saccades and reaction time on number of stimuli in the display. On the contrary, when two (or more) primitive features are conjoined in each stimulus, number of saccades and reaction time become regressively proportional to number of stimuli. Furthermore, a nearly 2:1 ratio is presumed to appear in the corresponding slopes for cases in which the target is absent rather than present, which may be regarded as diagnostic of a self-terminating serial processing, assuming that inspected items are permanently excluded from subsequent inspections. ${ }^{4}$

GST began as a modification of the basic form of FIT that we have just described (Wolfe, 1994). Preattentive processes and a corresponding activation map were emphasized, combining bottom-up (similarities among items) and top-down (target specifications) information, which sets feature and conjunction searches along a continuum. What differs across visual search tasks is the effectiveness of preattentive processes at guiding the attentional focus. Treisman and Gormican (1988) had similarly refined the original FIT by allowing features to be more or less coarsely coded or inhibited if they were shared less or more with the target, thus expanding or contracting correspondingly the size of the attentional focus. We refer to D. E. Williams and Reingold (2001) for further review and confirmation of GST versus early versions of FIT (but see Zelinsky, 1996).

The characterization of an activation map and its computational implementation in GST (Cave \& Wolfe, 1990; Wolfe, 1994) has been prominently paralleled by the development of a similar saliency map in a computational model 
of visual attention further mimicking its underlying neural circuitry (Itti \& Koch, 2000, 2001; Koch \& Ullman, 1985). As an alternative to item-based activation maps, a smoother area-based activation map has also been proposed and computationally implemented (Pomplun, Reingold, \& Shen, 2003). Its purpose is to extend GST so as to better account for distractor grouping effects that figure more prominently in the AET of Duncan and Humphreys $(1989,1992)$.

GST, in particular, depends on the signal detection problem, meaning that "the efficiency of search is determined by the strength of the attention-attracting signal of the target item relative to the attention-attracting noise of the distractors" (Wolfe, 1994, p. 210). SDT has independently evolved on the basis of developments in physiology and psychophysics in characterizing the mechanisms by which attention can influence visual search (Cameron, Tai, Eckstein, \& Carrasco, 2004; Green \& Swets, 1966; Palmer, Verghese, \& Pavel, 2000; Swets, 1964; Tanner \& Swets, 1954). Here, we can only refer to wider reviews and applications of SDT in visual search by Eckstein et al. (2000) and Verghese (2001).

\section{Our Study}

We designed a visual search task in which color and orientation of the stimuli are the only two separable features that must be integrated into conjunctive wholes in order to identify a target, according to basic FIT and its terminology. That task and the psychophysical measurements that we correspondingly performed (Keech, 2006; Keech et al., 2009) were relatively similar to those in the main experiment in Motter and Belky (1998a, 1998b), but with a major difference regarding participants. Namely, whereas Motter and Belky used 2 rhesus monkeys as their subjects, we worked with 14 human participants. Furthermore, whereas their monkeys were highly trained and practiced with the visual search task, our human participants were much less so. In addition, we studied separately two age groups - namely, 7 young adults with an average age of 20.4 years and 7 older adults with an average age of 49.7 years. Part of our project was to investigate how robust the Motter-Belky results should be regarded to be with respect to relatively minor differences in design, while determining which more substantial differences may exist between highly trained monkeys and relatively untrained humans of different ages.

We extensively reproduced and confirmed results of Motter and Belky (1998a, 1998b) regarding the following points. (1) Recognition of the target typically occurs with high probability only within a restricted area surrounding the current fixation location. The size of this zone of attentional focus, which we call AC, following Engel (1971, 1974, 1977), decreases with increasing stimulus density. (2) If the target is not detected within the current AC, a saccade is made most likely to an item just beyond that AC. That item has most likely the same color as the target, rather than the same orientation. (3) Except for those constraints, the item and new fixation point are selected apparently at random. (4) A forward bias is nevertheless apparent in consecutive saccades.
However, a survivor function analysis of cumulative probabilities of target detection revealed that our human participants made much greater use of memory than did Motter and Belky's (1998a, 1998b) monkeys, a result that we further confirmed by an analysis of reinspection rates and extensive computer simulations of visual search conduct (Keech, 2006; Keech et al., 2009; Keech \& Resca, in press). We shall thus maintain and depend on all those critical findings (regarding the role of visuospatial attention and memory in guiding eye movements and fixations) for the analysis and interpretation of the eye movement trajectories that we study more specifically in this article.

Here, statistical autocorrelation functions of the saccade direction cosines and their corresponding power spectra are studied first, in order to evaluate the contributions of memory, both in short-range form, such as a forward bias or IOR with limited capacity, plus the effects of the display boundary in turning eye movement trajectories, and in long-range form, such as a high-capacity but lowresolution memory for the search path. Then certain simple measures of circulation $(C)$ and imbalance $(I)$ in the eye trajectories are defined and statistically related to the corresponding subtended area $(A)$ on the display. These measures and their correlations are introduced and designed to reveal and characterize patterns in the eye movements that are characteristic of the search conduct, quite apart from psychophysical characteristics of saccades and fixations, such as saccade amplitude distributions and fixation duration distributions, which may underlie the search conduct but tell us nothing about those patterns. ${ }^{5}$

Our analysis ultimately demonstrates a prevalence of spiraling or circulating patterns over less organized eye trajectories. Three psychoneural mechanisms and two geometricspatial constraints are identified as primarily responsible for these pattern formations. The psychoneural mechanisms are (1) likelihood to saccade just beyond the current AC, (2) bias to saccade in a forward direction, possibly resulting from IOR with limited memory capacity, and (3) highcapacity but low-resolution memory for the search path. The geometric-spatial constraints are (4) search starting at the display center and (5) search confinement within the rectangular display boundary. Beyond that, no compelling evidence is found to require other interpretations in terms of either predetermined scanpaths or complex systematic search strategies, either common to all participants or idiosyncratically associated with each individual. On the other hand, alternative terminologies and conceptualizations of underlying mechanisms and their possible consequences can and should never be categorically excluded. We shall occasionally mention some such possible alternatives, while trying always to maintain a clear distinction between the facts that emerge from our data and analyses and the interpretations of those facts that we suggest or prefer.

\section{METHODS}

The participants in our experiments (Keech, 2006; Keech et al., 2009) were 7 young adults, 18-28 years of age, with an average age of 20.4 years, and 7 older adults, 
42-55 years of age, with an average age of 49.7 years. They wore a headpiece holding an Applied Science Laboratories Model 501 eyetracker and a magnetic head tracker. They viewed a 17 -in. Macintosh video display with effective dimensions of $11.25 \times 7.5 \mathrm{in}$. Within that display, search stimuli appeared as randomly distributed across a field of view of about $24.4^{\circ} \times 16.3^{\circ}$.

Our participants searched for a target bar that was randomly placed among $12,24,48$, or 96 additional distractor bars, in 90 trials for each array size. Interspersed randomly among those, there were 9 additional trials for each array size in which the target was absent. The participants were not informed of the number of target-absent trials. ${ }^{6}$ Before starting the actual experiment, each participant practiced briefly for 20 trials, comprising 5 trials for each array size.

The target was characterized by a unique conjunction of color and orientation: black bar, tilted $45^{\circ}$ to the right. Half of the distractors shared color with the target but had a different orientation (tilted $45^{\circ}$ to the left), whereas half of the distractors shared orientation with the target but differed in color (red). The participants were instructed to fixate a cross appearing at the center of the display at the beginning of each trial, but there were no further instructions or restrictions regarding any subsequent eye movement or fixation. The participants were required to make a speeded search decision about the presence or absence of the target in the array by pressing one of two buttons on a keypad with their left or right hand, respectively. The search array remained on for $4.0 \mathrm{sec}$ or until a response to the search task was made.

In order to analyze statistically the observed eye movements and reveal their patterns and underlying mechanisms, we introduce in this article certain variables and measures that can be treated with standard tools of stochastic and spectral analysis. We consider, for example, autocorrelation functions and their discrete Fourier transforms or power spectra. Basic concepts are summarized in the following section, and some mathematical details needed for our applications are discussed in Appendix A. More extensive treatment and technical details can readily be found in the standard literature on this subject (e.g., Oppenheim, Schafer, \& Buck, 1999). Then, in the subsequent section, we consider discrete distributions of certain measures of circulation and imbalance in the eye trajectories. We also consider scatterplots of circulation with a measure of the area subtended by the eye trajectory on the display. Standard methods of statistical analysis are applied to those measures and their relations, with further illustrations reported in Appendix B.

\section{SACCADE DIRECTION COSINE AUTOCORRELATIONS AND POWER SPECTRA}

The autocorrelation function of a variable that fluctuates in time is generally defined as a statistical or ensemble average of a product of two values of that variable attained at two given points in time. The ensemble average elimi- nates the random component in the relative fluctuations of those two values. The systematic component that survives statistical averaging of the product reveals the real measure of self-correlation, or "memory," that persists within the fluctuating variable with the passing of time.

In our application, we consider the two-dimensional saccade velocity as projected on the display, $\mathbf{v}(t)$, and its scalar or dot product at two points in time - that is,

$$
\begin{aligned}
\mathbf{v}\left(t_{1}\right) \cdot \mathbf{v}\left(t_{2}\right) & =v_{x}\left(t_{1}\right) v_{x}\left(t_{2}\right)+v_{y}\left(t_{1}\right) v_{y}\left(t_{2}\right) \\
& =\left|\mathbf{v}\left(t_{1}\right)\right|\left|\mathbf{v}\left(t_{2}\right)\right| \cos \theta\left(t_{1}, t_{2}\right) .
\end{aligned}
$$

Then we consider the statistical average

$$
K\left(t_{1}, t_{2}\right)=\left\langle\mathbf{v}\left(t_{1}\right) \cdot \mathbf{v}\left(t_{2}\right)\right\rangle
$$

over a sample of trials. That can be expressed equivalently in terms of the time interval $\tau=t_{2}-t_{1}$ between $t_{1}$ and $t_{2}-$ that is,

$$
K\left(t_{1}, t_{2}\right)=K\left(t_{1}, t_{1}+\tau\right)=\left\langle\mathbf{v}\left(t_{1}\right) \cdot \mathbf{v}\left(t_{1}+\tau\right)\right\rangle .
$$

An autocorrelation function is also called, figuratively, a memory function, because it refers to how much a fluctuating mathematical function - for example, a velocity $\mathbf{v}\left(t_{2}\right)$ - may "remember" beyond chance of what value it carried at a previous time [e.g., $\left.\mathbf{v}\left(t_{1}\right)\right]$. Of course, this represents merely the conventional terminology generally adopted in mathematics and statistical physics (cf. Pathria, 1996). Interestingly, in our application this "anthropomorphic" connotation of the autocorrelation, or memory function, can be interpreted somewhat more literally, because it does ultimately derive from psychoneural mechanisms of mnemonic, perceptual, and attentional origins. That would certainly not be the case if we were talking about autocorrelation functions of Brownian particle velocities due to molecular bombardment, for example.

Since we are mostly interested in the directional patterns of the eye trajectories, we divide each velocity dotproduct by the product of the two velocity magnitudes, before computing the sample average. Furthermore, since a search trial consists of a sequence of saccades, we regard time as merely an integer counting of the saccades in the trial; that is, we set $\tau=l=0,1,2,3, \ldots$ The resulting autocorrelation function, denoted as $R(l)$, thus represents the statistical average of the cosine of the angle between saccade directions separated by an integer number $l$ of intervening saccades in a trial or trajectory - that is,

$$
R(l)=\left\langle\cos \theta\left(t_{1}, t_{2}\right)\right\rangle .
$$

This choice has certain advantages over various alternatives, such as that of averaging directly the angle $\theta\left(t_{1}, t_{2}\right)$ between saccade directions at different times. For angles ranging from $-180^{\circ}$ to $+180^{\circ}$, the cosine is an even function, monotonically decreasing from +1 to -1 for either positive or negative angles. That resolves the modulo ambiguity inherent to angular variables (such that a turn of $175^{\circ}$ to the left may also have been a turn of $185^{\circ}$ to the right, e.g.), in addition to providing more convenient units. Furthermore, if there is no intrinsic bias to saccade either 
to the left or to the right, which we will demonstrate by a complementary study of the saccade circulation, $\theta\left(t_{1}, t_{2}\right)$ itself should average to zero invariably, prompting to average an even function of $\theta\left(t_{1}, t_{2}\right)$ in any case. For those two reasons in particular - that is, the resolution of the modulo ambiguity and the convenience of units - we found it to be preferable to study the autocorrelation $R(l)$ of the cosine of $\theta\left(t_{1}, t_{2}\right)$, rather than the autocorrelation of the magnitude of $\theta\left(t_{1}, t_{2}\right)$, for example, even though the cosine function, being nonlinear, slightly overweights forward and backward directions, as compared with the magnitude of $\theta\left(t_{1}, t_{2}\right)$. Further details on some mathematical and statistical properties of autocorrelation functions and their numerical treatment in our application are reported in Appendix A.

A

12-Distractor Array

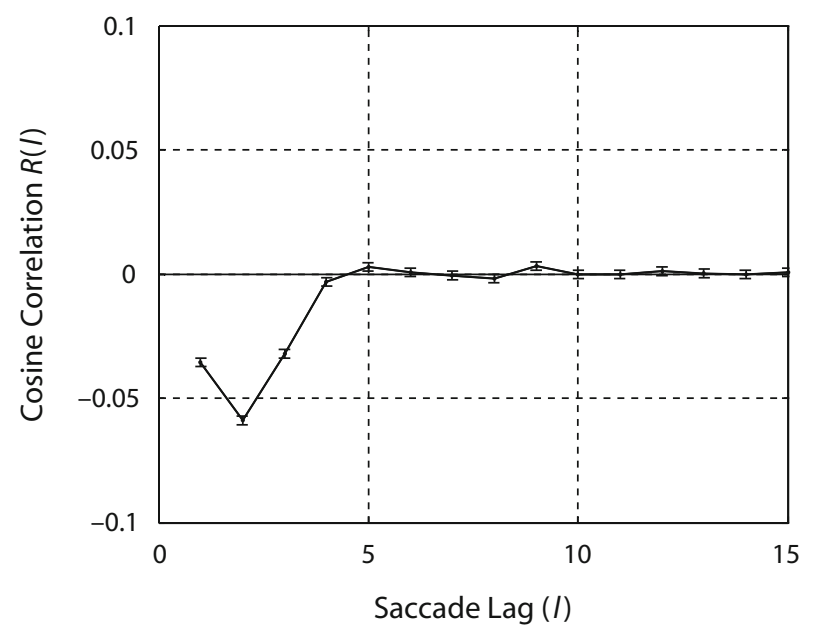

C 48-Distractor Array

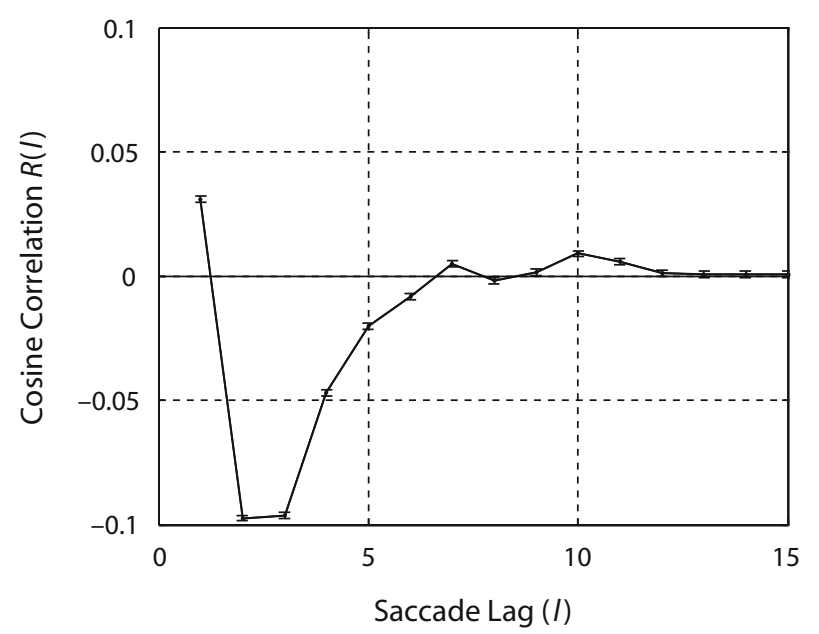

Figures 1A-1D show the saccade direction cosine autocorrelations $R(l)$ for the older adults searching arrays of each size. The corresponding results for young adults are similar (Keech, 2006). ${ }^{7}$ The maximum value $\cos (0)=1$ can survive after sample averaging only for the same saccade autocorrelation - that is, only for $R(l=0)=$ $\left\langle\cos \theta\left(t_{1}=t_{2}\right)\right\rangle=1$. That initial value is understood and omitted from Figures $1 \mathrm{~A}-1 \mathrm{D}$, in order to expand the ordinate scale and display the small standard error bars estimated in Appendix A.

The 48- and 96-distractor autocorrelations are clearly characterized by slightly positive $R(l=1)$ values (for older as well as young adults). This derives mainly from a tendency of consecutive saccades to progress in a forward direction, which has been noted as a forward bias in vari-
B

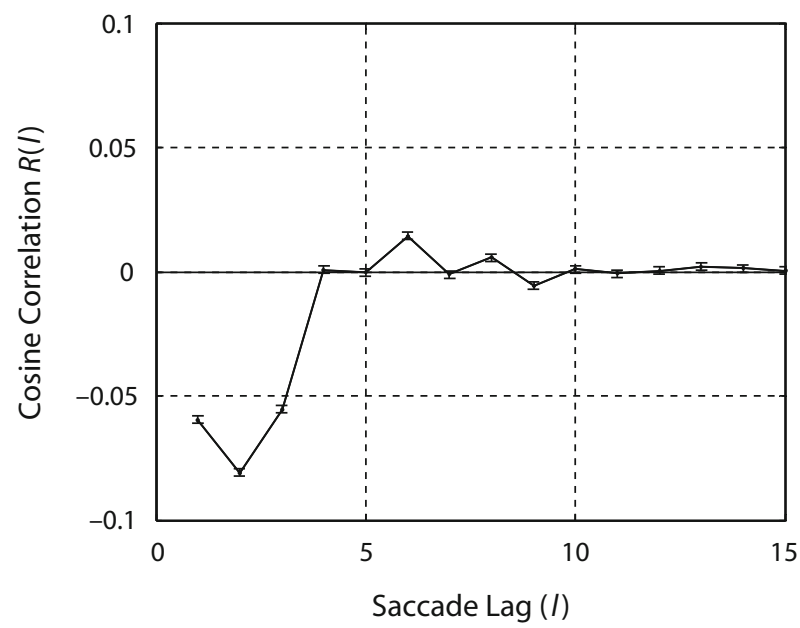

D 96-Distractor Array

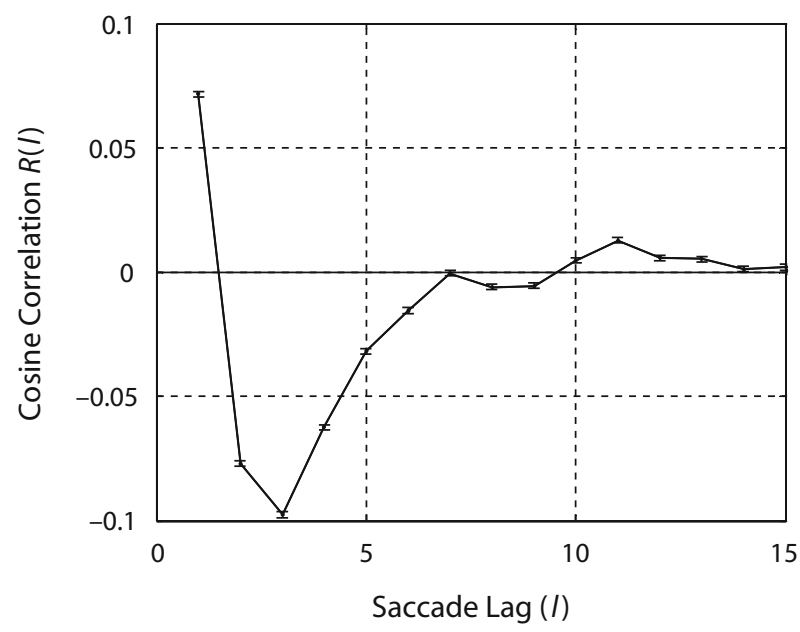

Figure 1. Saccade direction cosine autocorrelations $R(l)$ for older adults searching arrays with 12 (A), 24 (B), 48 (C), and 96 (D) distractors. Standard error bars are displayed with $\pm \mathbf{0 . 0 0 1 7}, \pm \mathbf{0 . 0 0 1 5}, \pm \mathbf{0 . 0 0 1 2}$, and \pm 0.0011 relative values for 12-, 24-, 48-, and 96-distractor arrays, respectively. The integer $l=0,1,2,3, \ldots, 15$ in abscissa labels the lag or number of intervening saccades between the correlated pair. The initial value $R(0)=1$, representing the autocorrelation of each saccade with itself, is out of scale and omitted. Lines joining adjacent $R(l)$ values are added merely as a guide to the eye. 
ous previous observations. For example, the scatterplot in Figure 11 in Keech et al. (2009) shows a greater concentration of angle shifts in the $0^{\circ}-90^{\circ}$ and in the $270^{\circ}-360^{\circ}$ ranges for cases in which the second saccade has an amplitude of less than about $7^{\circ}$. Figure 10B in Keech et al. (2009) and Figure 7B in Motter and Belky (1998a) show the same forward bias in another type of scatterplot.

For $l=2,3, R(l)$ becomes negative for all display sizes, indicating that, starting from any given saccade, the second and the third saccades tend to form angles between $90^{\circ}$ and $270^{\circ}$ relative to the original saccade, on average. This can be caused only by the boundary of the display, which forces saccades to turn around, thus overcoming their forward bias. ${ }^{8}$ In fact, for 12- and 24-distractor arrays, even the $R(l=1)$ values have already turned negative. This occurs because "bouncing back" at the boundary immediately after the initial saccade from the display center occurs more frequently in the smaller arrays, where the search is also more likely to terminate promptly, whereas, in the larger arrays, bouncing of consecutive saccades around the display periphery permits more manifestation of their forward bias in $R(l) .{ }^{9}$ The scatterplot in Figure 11 in Keech et al. (2009) shows independently the direction reversal in consecutive saccades induced by the display boundary when the second saccade amplitude exceeds about $7^{\circ}$.

Advancing further, the autocorrelation $R(l)$ drops substantially in magnitude beyond $l=6$. This means that distributions of direction cosines between saccades separated by more than six saccades become relatively random, although not completely so until much later, particularly for the larger arrays. For 12-distractor arrays, the autocorrelation $R(l)$ becomes relatively random only after $l=3$.

Figures 2A and 2B show discrete power spectra for the older adults searching arrays with 12 and 96 distrac-

A

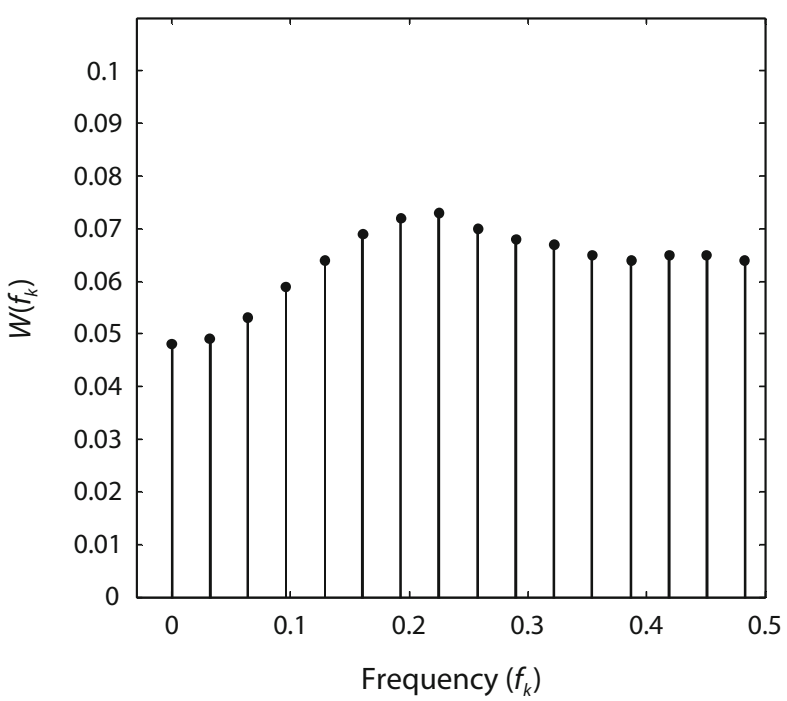

tors, respectively. The more any power spectrum deviates from a constant, or white noise, the more it is generally associated with "memory," in a conventional and figurative sense of these words (cf. comments after Equation 2). Correspondingly, the autocorrelation, or memory function, increasingly deviates from its baseline $B(l)=\delta_{l, 0}$ for pure chance. The power spectrum in Figure 2B clearly deviates from white noise considerably more than does the power spectrum in Figure 2A, indicating more "memory" overall. The specific shape of a power spectrum provides additional information about the form of memory that underlies the dynamics of the system. According to a common rule of thumb explained in Appendix A, relatively large components $W\left(f_{k}\right)$ of the power spectrum at high frequencies $f_{k}$ correspond to appreciable components of the autocorrelation $R(l)$ at short range $l$, and conversely at low frequencies. This relation, which is typical of Fourier transforms, is expressed in our case and notations as $l \sim 1 / f_{k}$ for the appreciable and dominant components of $R(l)$ and $W\left(f_{k}\right)$, respectively. Having set $f_{k}=k /(2 L-1)$ and $L=16$ in our application, we have, correspondingly, $l \sim 31 / k$. Further discussion and details on these matters are provided in Appendix A.

In Figure 2B for 96-distractor arrays, the dominant components of $W(k)$ occur at $k=3,4,5,6$. Those correspond to $l \sim 10,8,6,5$, indicating a correlation length of at least 8 to 10 intervening saccades for an average trajectory. That length is consistent with the direct observation of appreciably nonvanishing components of $R(l)$ in Figure 1D. These results thus suggest the presence of a long-range memory component, attributable to some psychoneural mechanism. That would be consistent with a high-capacity but low-resolution form of memory for the search path suggested by Dickinson and Zelinsky (2007) in particular.

\section{B}

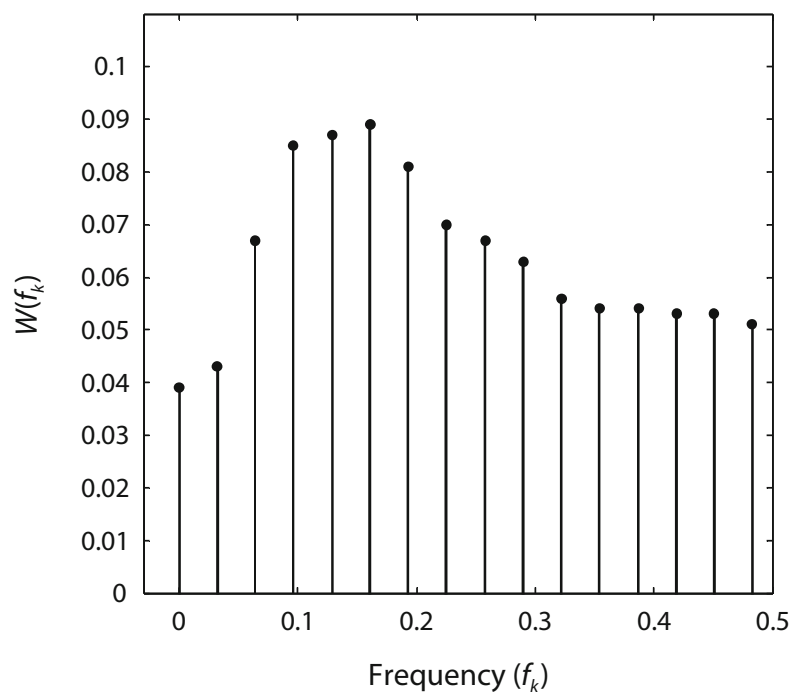

Figure 2. Discrete power spectra $W\left(f_{k}\right)$ of the saccade direction cosine autocorrelations $R(l)$ for the older adults searching arrays with 12 (A) and 96 (B) distractors. 
In Figure 2A for 12-distractor arrays, $W(k)$ is much less peaked, with a lower maximum at a greater $k=7$. That corresponds to $l \sim 4$ and is consistent with the small magnitude of $R(l)$ beyond $l=3$ in Figure 1A. Apparently, trajectories in 12-distractor arrays have a lesser chance of experiencing long-range memory effects, mainly because there are fewer saccades in those trajectories, on average. ${ }^{10}$ Vice versa, $W(k)$ retains almost maximal components all the way up to the largest $k=15$ in Figure 2A. That corresponds to $l \sim 2$, which indicates a greater effect of shortrange memory, as compared with the case of Figure 2B for 96-distractor arrays. What counts effectively as a form of short-range "memory" is mainly the countering of the forward bias, which affects consecutive saccades $(l=1)$, by the boundary constraint, which causes saccades to "bounce" back or around after reaching the screen periphery $(l=1,2,3)$, as already indicated by the corresponding $R(l)$ values in Figure 1.11 This form of short-range "memory," corresponding to power at high frequencies, operates more effectively in smaller than in larger arrays mainly because more trajectories reach earlier the display boundary in lower density arrays.

Further insight derives from the comparison of Figures 1 and 2 with Figures B6A and B6B, which show $R(l)$ and $W(k)$ for 60,000 random-walk simulations with parameters corresponding to 96-distractor arrays searched by the older adults, including the rectangular boundary constraint. These random-walk simulations stay clear of any psychoneural element, of course. The corresponding elimination of long-range memory (for the search path) is manifested by the drastic relative reduction in power at low frequencies in Figure B6B, as compared with both Figures $2 \mathrm{~A}$ and $2 \mathrm{~B}$. Forward bias is also eliminated, but the effect of the boundary constraint remains. That implies retention of an effective form of short-range memory (not psychoneural, but in the mathematical sense). Correspondingly large high-frequency components of $W(k)$, relative to its small low-frequency components, are observed in Figure B6B. Now, if we remove even the boundary constraint and perform completely random-walk simulations, we expect virtually identical components of $W(k)$ at all frequencies, which is indeed what we observe in Figure B7B, representing white noise.

Power spectra of dynamical systems can be further analyzed in order to reveal more specific types of noise and memory structures. Particularly interesting applications to visual search have been provided by Aks et al. (2002), Boccignone and Ferraro (2004), Brockmann and Geisel (2000), and Najemnik and Geisler (2005). All these studies are reviewed in greater detail in a companion article of ours (Keech \& Resca, in press). At this point, we develop instead an alternative approach, by devising different criteria and measures that apply directly to the eye movement trajectories and further characterize their patterns. That complements the autocorrelation and power spectra results in identifying the basic psychoneural mechanisms that adapt and combine with the geometrical and spatial constraints of the task so as to make the visual search most effective.

\section{CIRCULATION, IMBALANCE, AND AREA MEASURES}

A simple measure that can be used to characterize the eye movement trajectories is the circulation index $C$ defined as follows. Scanning clockwise or counterclockwise, the angle shift from one saccade to the next one is either to the right or to the left, which we score as a +1 or a -1 , respectively. Progressing along the search trajectory, those units are added algebraically to compute the total $C$. Thus, a mainly clockwise or counterclockwise trajectory produces a value of $C$ relatively large in magnitude, whereas a more disorganized or random path typically generates a $C$ smaller in magnitude.

On the other hand, it is possible for a trajectory to attain a relatively large value of $C$ in magnitude without actually covering a large area in the display. In order to estimate the likelihood of that happening, and much more, we further consider the maximum and minimum values of the horizontal and vertical fixation coordinates attained throughout a trajectory. By taking the product of the differences $\Delta X$ and $\Delta Y$ between those maxima and minima, we define a measure of the area spanned by the trajectory-namely, $A=\Delta X \cdot \Delta Y .^{12}$ Thus, $A$ is the area of the rectangular box that covers most tightly (and without tilting) any given trajectory.

Evidently, for a (rare) trajectory with a single saccade, $A$ is a rather meaningless estimate, varying between zero and half the square of the saccade length, depending on the saccade orientation. However, as the number of saccades increases in a trajectory, the overall trajectory orientation within the rectangular display and coordinates becomes less relevant, and $A$ begins to provide a still crude but more meaningful estimate of the display area actually spanned by the trajectory. The lengths of the individual saccades within the trajectory also become less relevant, since those lengths vary stochastically according to a well-defined saccade amplitude distribution (e.g., as shown in Figure 7 of Keech et al., 2009). Finally, when many trajectories are treated statistically, individual peculiarities further disappear, and the study of the statistical correlation in the scatterplots between $A$ and $C$, which is ultimately what matters to us, ends up revealing the underlying patterns more characteristic of the eye trajectories. In turn, that indicates the mechanisms that guide and constrain the visual search.

The discretized distribution of the circulation $C$ and its scatterplot with the area measure $A$ are plotted in Figures 3-6 for the 12-, 24-, 48-, and 96-distractor arrays searched by the older adults. Corresponding figures for young adults are similar (Keech, 2006). The most notable features of these graphs are the following. (1) The $C$ distributions are typically bimodal and symmetric about $C=0$. This suggests a prevalence of spiraling or circulating eye trajectories, equally likely to develop in either the clockwise or the counterclockwise direction. (2) As the array size increases, the $C$ distribution broadens and its peak values decrease. This is mainly a consequence of the increasing average number of saccades in the larger arrays, which increases the maximum magnitude of $C$. (3) The 
A

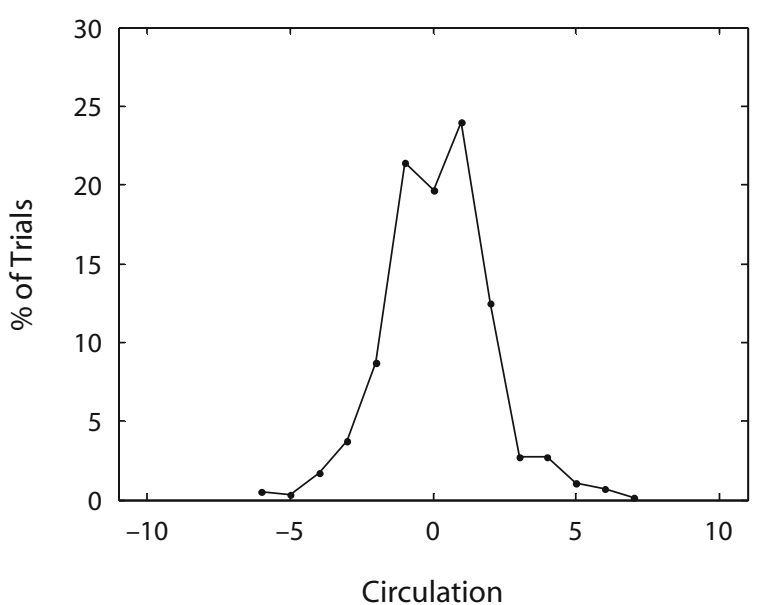

B

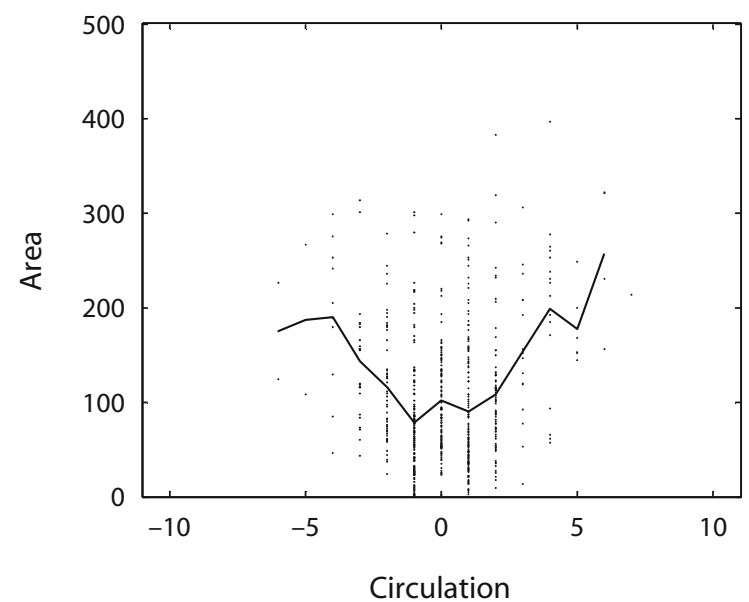

Figure 3. Circulation $C$ discretized distribution (A) and its scatterplot with the area measure $A(B)$ for the older adults searching 12-distractor arrays. The integer values $C$ of possible circulation are reported in the abscissas. In panel $A$, the percentages of trials or trajectories with the corresponding $C$ are reported in the ordinates. Those ordinates are then connected with line segments merely as a guide to the eye. In panel $\mathrm{B}$, a solid line is drawn to connect the average values of $A$ at different $C$ s. All types of trials, with or without target addition, are considered.

A

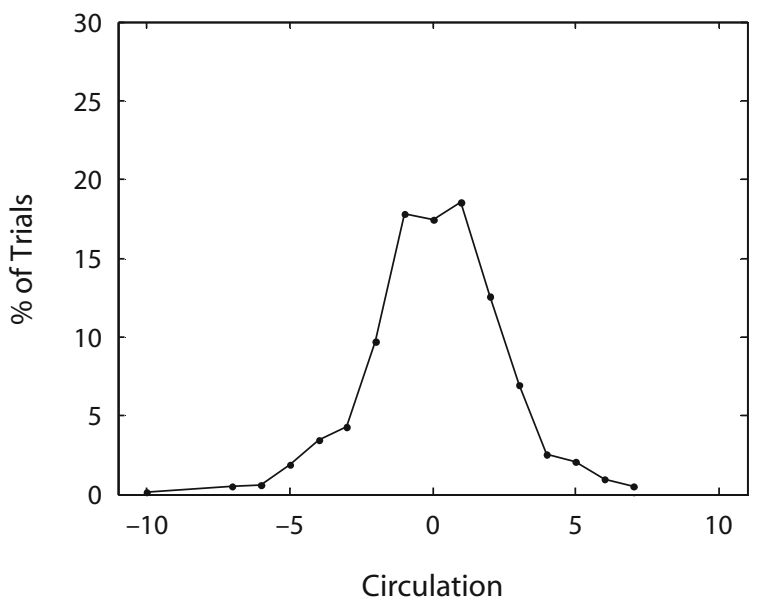

B A-C Scatterplot

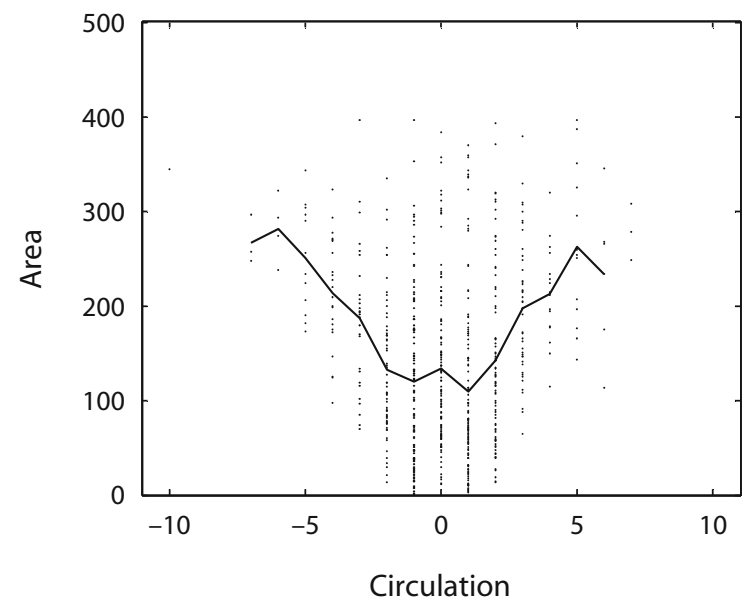

Figure 4. Circulation $C$ distribution (A) and its scatterplot with the area measure $A(B)$ for the older adults searching 24-distractor arrays.

average value of $A$ increases curvilinearly with $|C|$. For example, a quadratic relation $A=6 C^{2}+100$ fits reasonably the scatterplot in Figure 3B for $|C|<5$. A curvilinear relation between $A$ and $C$ confirms the presence of spiraling or circulating eye trajectories. Further appreciation and understanding of these features derives from the study of opposite limits of perfectly circular trajectories and random walks, which are discussed in Appendix B.

Since the number of saccades increases with array size on average, so does the circulation magnitude, as noted in the previous Point 2. In order to make more direct comparisons between search arrays of different sizes, we may wish to consider a correspondingly "normalized" statistic - that is, the fraction of turns to either side of the trajectory. That can- not be inferred from the knowledge of the circulation alone, since it requires further knowledge of the total number of saccades following the first one, which, in turn, equals the number $V$ of vertices joining the saccade segments in the completed trajectory. For example, in a trial with 13 saccades, there is a number $V=12$ of angle shifts or vertices. If $C=+8$, then 10 angle shifts must have been to the right and 2 angle shifts must have been to the left. Thus, 10/12, or about $83 \%$, of the angle shifts went to the right. We define that fraction as the imbalance I in circulation. Thus, $I$ is basically the circulation magnitude "normalized" by the number $V$ of intervening saccades and counted above chance, which is set at $I=0.5$. Mathematically, the relation is thus expressed as $|C|=V \cdot(2 I-1)$. 
A

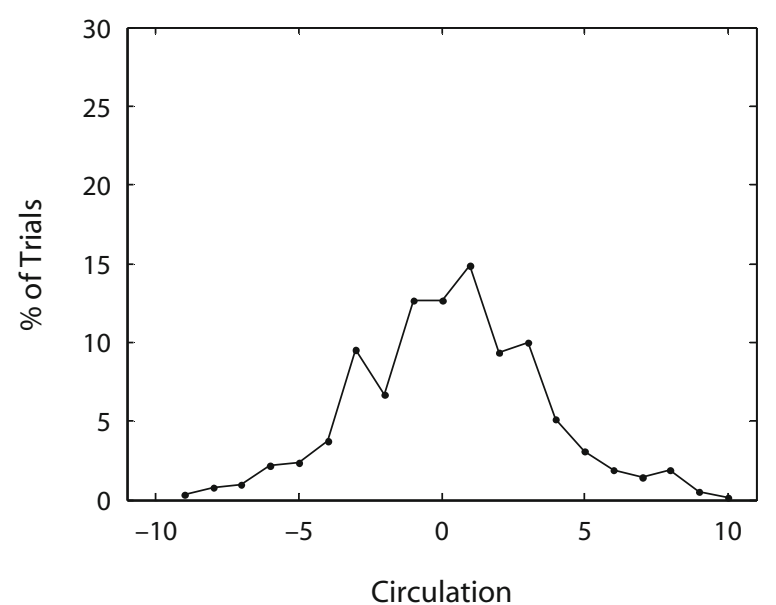

B A-C Scatterplot

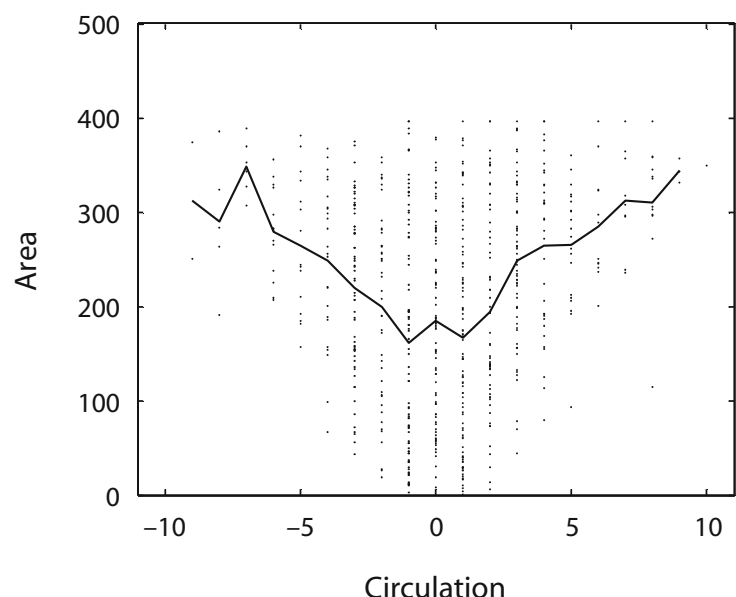

Figure 5. Circulation $C$ distribution (A) and its scatterplot with the area measure $A$ (B) for the older adults searching 48-distractor arrays.

A

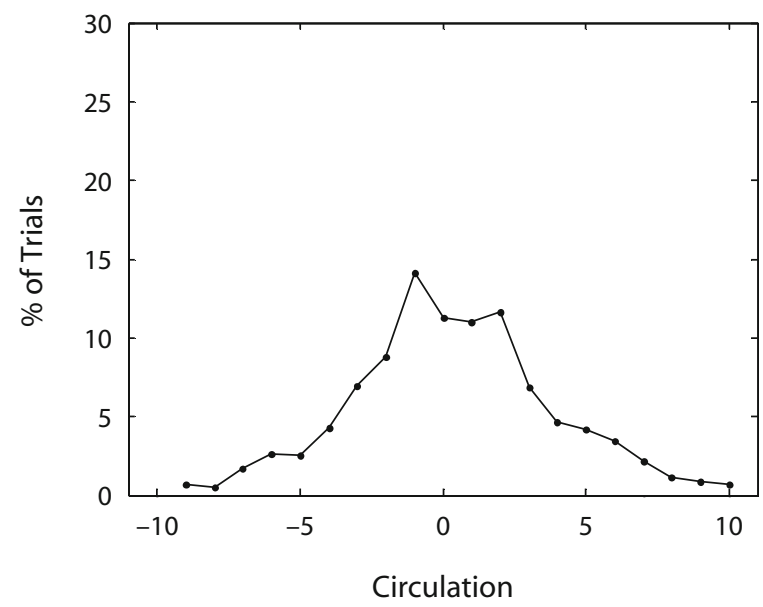

B A-C Scatterplot

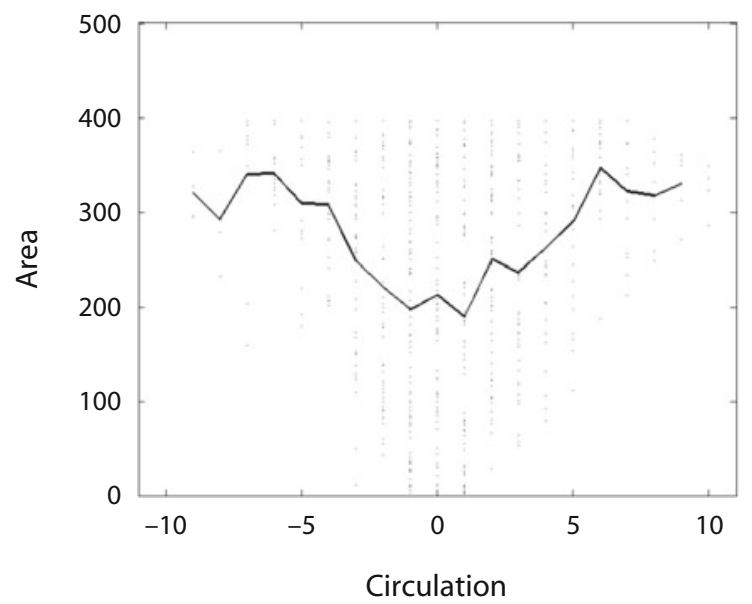

Figure 6. Circulation $C$ distribution (A) and its scatterplot with the area measure $A$ (B) for the older adults searching 96-distractor arrays.

Average values of $I$ are $81 \%, 80 \%, 75 \%$, and $70 \%$ for young adults searching 12-, 24-, 48-, and 96-distractor arrays. The corresponding average values of $I$ for the older adults are $79 \%, 77 \%, 73 \%$, and $70 \%$. The most striking feature that emerges immediately from these results is that, on average, as many as three out of four intervening saccades turn to the same side, basically regardless of search array size. Still, that does not imply either a clockwise or a counterclockwise bias in the trajectories overall, which is ruled out by the inversion symmetry of the $C$ distributions, as was already noted in the previous Point 1.

Imbalance discretized distributions for arrays of all sizes, searched by both young and older adults, are shown in Figures $7 \mathrm{~A}-7 \mathrm{D}$ and $8 \mathrm{~A}-8 \mathrm{D}$, respectively. Young adults show slightly (but not significantly) more imbalance than do older participants. The dominant peaks in all the imbalance distributions occur at $100 \%$ imbalance, or $I=1$, representing angle shifts all to the same side. Those peaks decrease as the array size increases, from a maximum of $49.7 \%$ of the trials in Figure 7A to a minimum of $16.8 \%$ of the trials in Figure 8D. This merely reflects that $I=1$ is more likely to occur in trials with fewer saccades. In fact, the average number of angle shifts associated with $I=1$ is only 1.8 , 2.0, 2.4, and 2.6, for 12-, 24-, 48-, and 96-distractor arrays searched by young adults, while their average number of angle shifts for any imbalance is $2.4,3.0,5.1$, and 6.8 , respectively. For the older adults, $I=1$ involves an average number of angle shifts (and a corresponding circulation magnitude) of 1.8, 2.1, 2.7, and 2.9 for 12-, 24-, 48-, and 96-distractor arrays, while their average number of angle shifts for any imbalance is $2.3,3.2,5.5$, and 7.4 , respectively. This indicates that the dominant peaks at $I=1$ in the 
A

12-Distractor Array

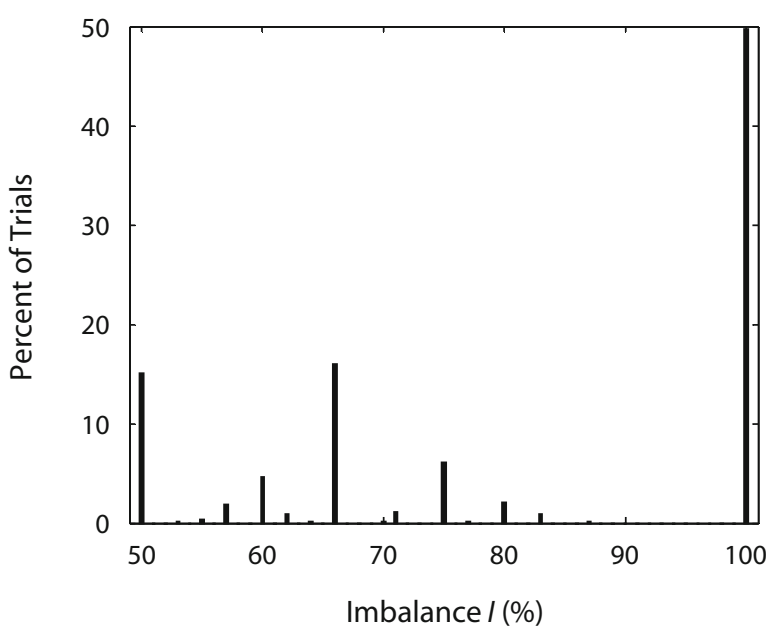

C

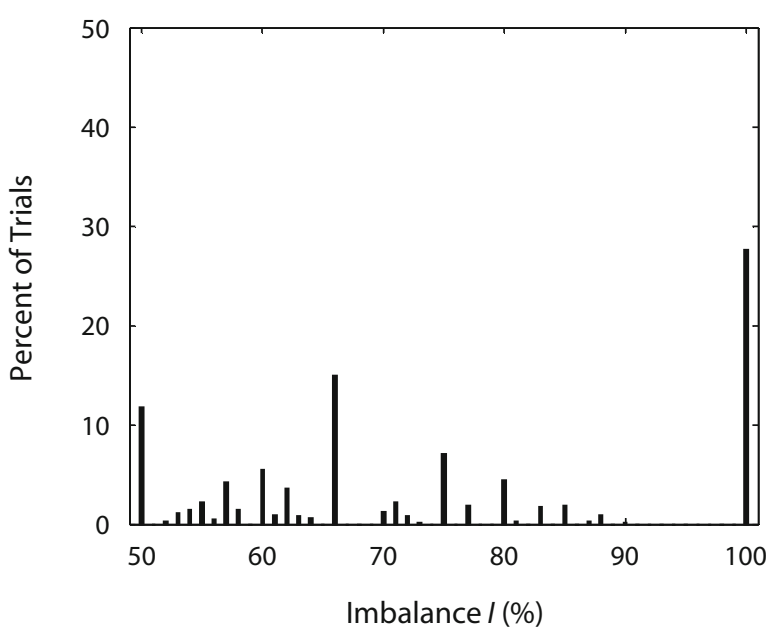

B

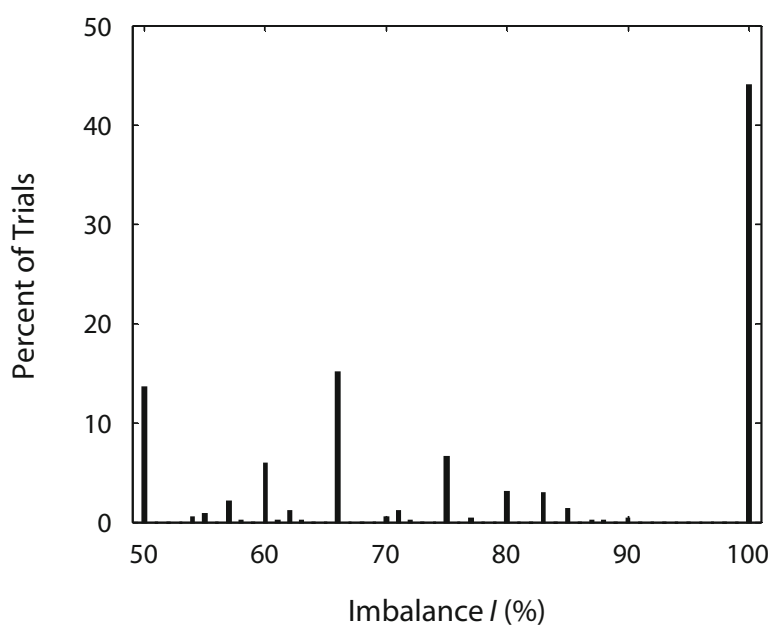

D

96-Distractor Array

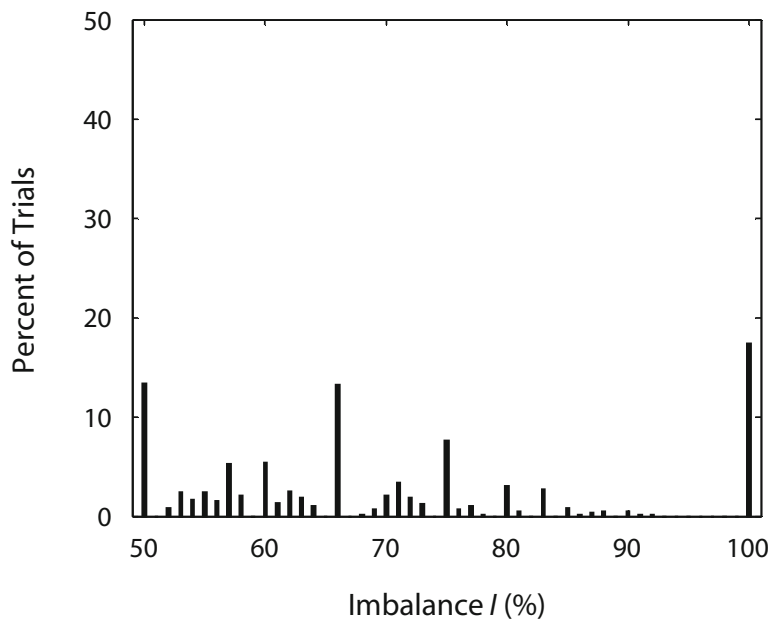

Figure 7. Imbalance I discretized distributions for young adults searching arrays with 12 (A), 24 (B), 48 (C), and 96 (D) distractors. The discrete values of possible imbalance percentages $I$ are reported in the abscissas, whereas the percentages of trials or trajectories with the corresponding $I$ are reported in the ordinates. All types of trials, with or without target addition, are considered.

imbalance distributions largely contribute to the bimodal peaks in the circulation distributions, particularly for the smaller array sizes. By contrast, $15.1 \%$ of trials, at most, have no imbalance $(I=0.5$ or $C=0)$ for all array sizes searched by young adults, rising at most to $19.7 \%$ of trials for all array sizes searched by the older adults. The fact that eye trajectories with $100 \%$ imbalance substantially outnumber (by ratios of at least 1.5 and as much as 3.3) eye trajectories with no imbalance provides further confirmation that spiraling or circulating patterns prevail over more disorganized or random patterns in the visual search.

\section{SCANNING PATTERNS}

For the purpose of illustration, we begin by showing in Figures 9A-9D four examples of 96-distractor arrays searched by visibly spiraling or circulating eye trajecto- ries, with their $C$ and $A$ correspondingly noted in the captions. In Figures 9A, 9B, and 9D, the participant was fixating sufficiently close to the target when the search ended. In both Figures 9B and 9C, the target fell within or close to an AC long before the search ended, but the participant failed to recognize the target at that time. In Figure 9C, the participant actually failed to recognize the target at any time during the search.

In Figures 10A-10D, we show four examples of 96-distractor arrays searched by apparently more disorganized or random eye trajectories. In Figure 10B, the target is absent. In Figures 10A and 10C, the search ends with a fixation near the target, confirming its correct recognition. In Figure 10D, the target was closest to, and probably detected in, the next-to-the-last fixation, but it was recognized only after an additional saccade elsewhere. We found that this occurrence is not so uncommon and could 
A

12-Distractor Array

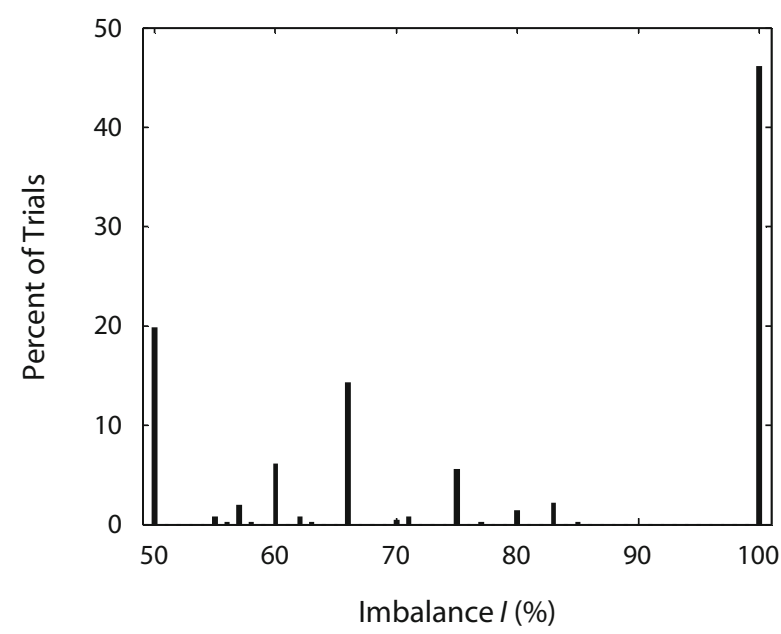

C 48-Distractor Array

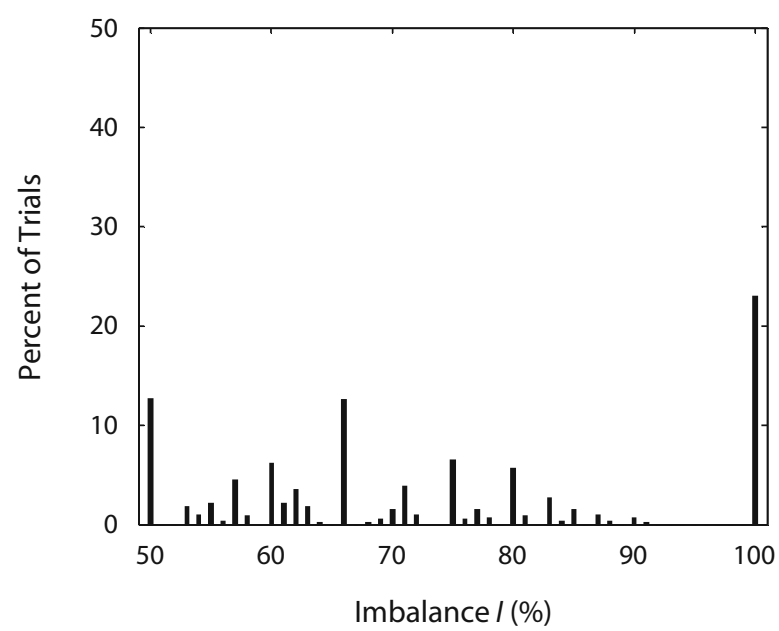

B

24-Distractor Array

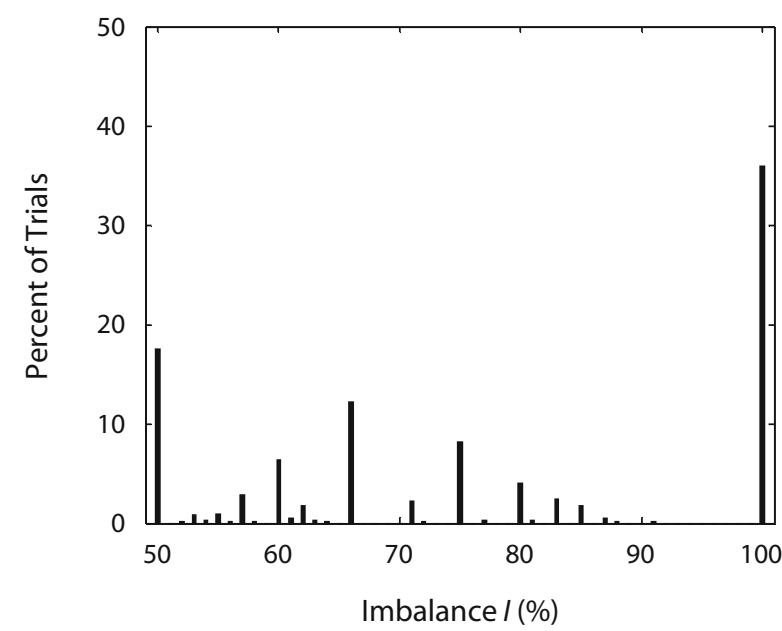

D 96-Distractor Array

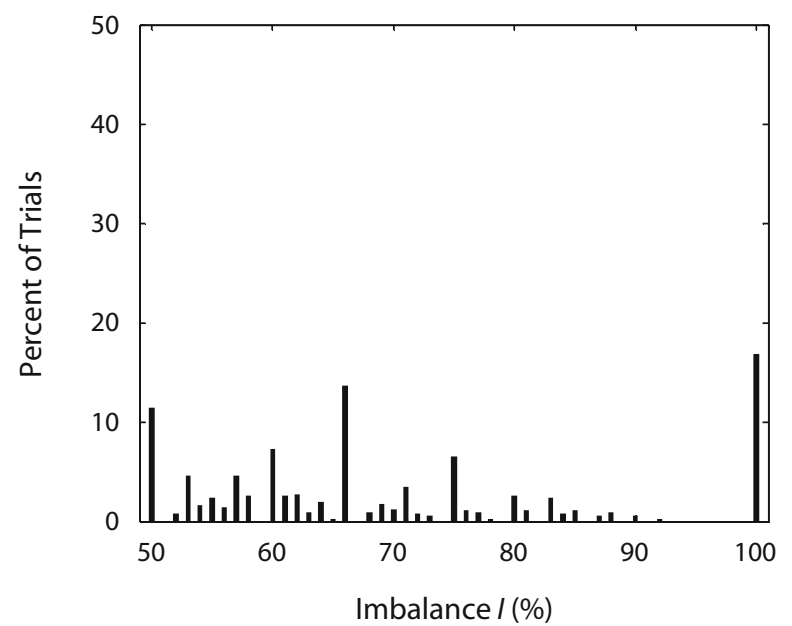

Figure 8. Imbalance $I$ discretized distributions for the older adults searching arrays with 12 (A), 24 (B), 48 (C), and 96 (D) distractors.

result either from a delay in target recognition (Engel, 1977; Hooge \& Erkelens, 1996, 1998; Motter \& Belky, 1998a, p. 1808) or its communication (by buttonpressing) or from programming in parallel of two consecutive saccades during some fixations (Findlay et al., 2001; McPeek et al., 2000). Figures 10A-10C represent less likely cases of a small $C$ that is nonetheless associated with a large $A$, which typically prevents a circulating pattern. In Figure 10D, the search ended with a rather fortuitous capture of the target after only four saccades. As a result, both $C$ and $A$ are small, and the pattern cannot be clearly classified as either circulating or random, since the trajectory did not develop long enough to tell. That is, of course, a more common occurrence in the smaller search arrays.

Incidentally, note throughout Figures 9 and 10 that participants tend to fixate closer to objects that share color with the target, rather than orientation, in agreement with the basic model and observations of Motter and Belky (1998a, 1998b) and ourselves (Keech, 2006; Keech et al., 2009, Figure 9). Color segmentation has indeed figured prominently in visual search theories (FIT, GST; Pashler, 1987), since its compelling demonstration by Egeth, Virzi, and Garbart (1984).

We visually inspected hundreds of search trajectories such as those shown in Figures 9 and 10, looking for hints of recurrence of more systematically structured patterns. We were looking for scanning strategies reported in other studies that used displays more structured than ours, such as scanning left to right, top to bottom, rasterlike, snakelike, hub and spokes, and so forth. We were unable to clearly identify any strategy of that sort. Motter and Belky (1998a) had similarly concluded that "the pattern observed on any given trial seemed to arise as a function of the distribution of stimuli having the target color within the boundaries of the display, rather than any scanning strategy" (p. 1810). The only usually recognizable pattern 
A

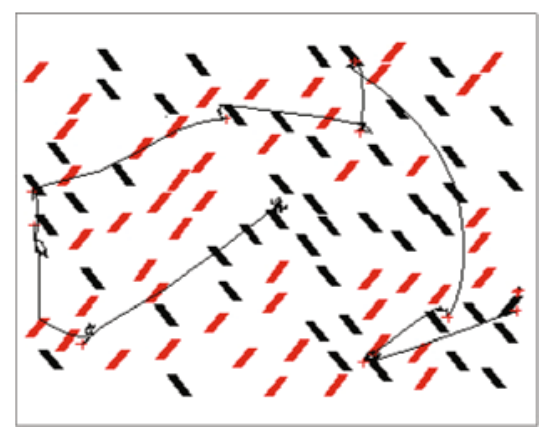

C

$$
C=-6, A=234
$$

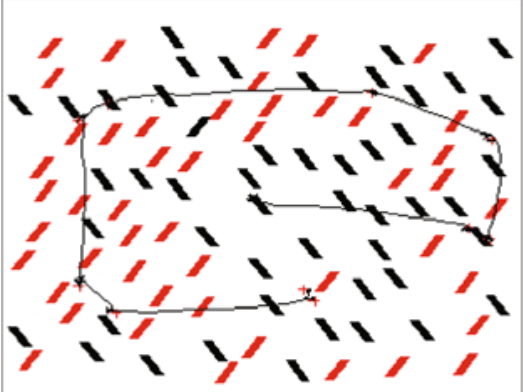

B

$$
C=6, A=350
$$

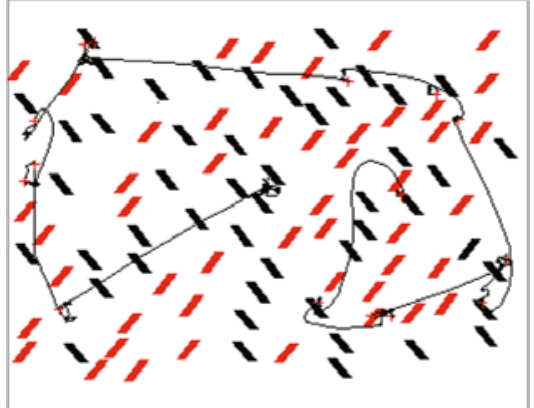

D

$$
C=7, A=286
$$

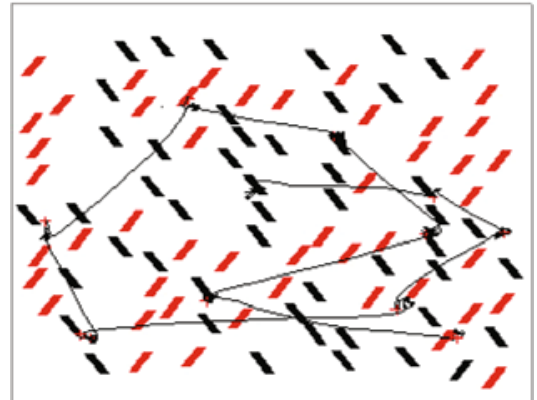

Figure 9. Search trajectories suggestive of spiraling or circulating patterns.

A

$$
C=0, A=319
$$

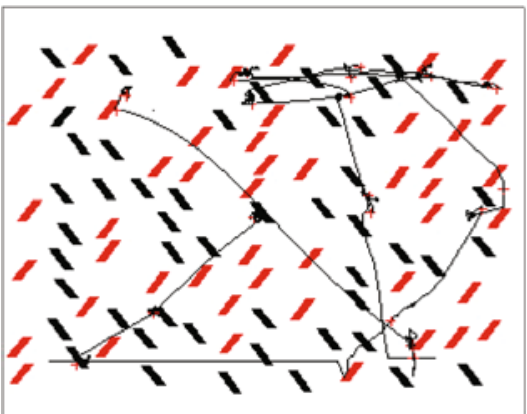

C

$$
C=-1, A=361
$$

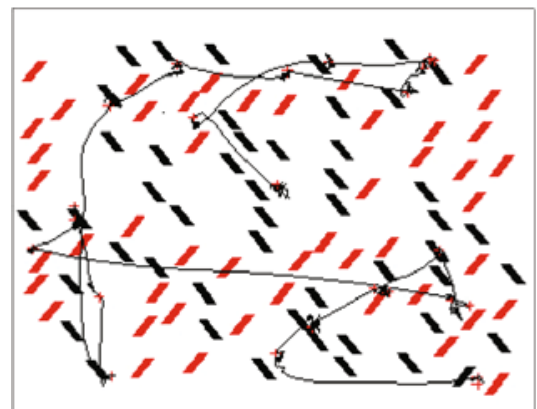

B

$$
C=2, A=314
$$

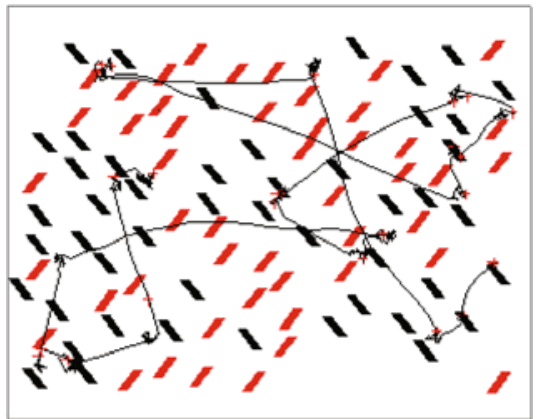

D

$$
C=-1, A=121
$$

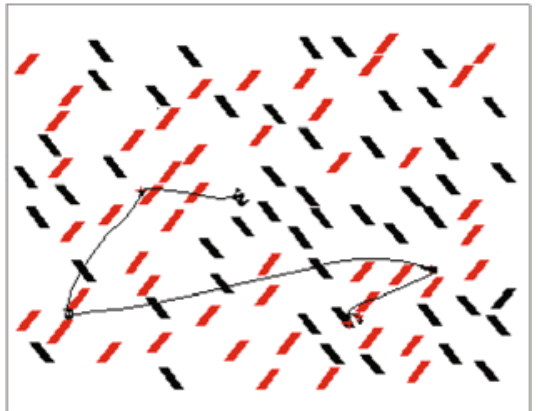

Figure 10. Apparently more disorganized or random search trajectories. 
in our experiments is that of spiraling out from the screen center and then more or less bouncing around the screen periphery, as in Figures 9A-9D, for example. To a certain extent, that results from the physical conditions in our task and displays, where the search must start at the screen center and the target is placed randomly and uniformly within the boundaries of the display. These conditions make it more likely that the target is captured either on the way outward from the initial central fixation or while circulating around the screen periphery, rather than on any return inward from the periphery, since the latter could occur only if the target had not been captured thus far, which is less likely. Indeed, the fact that the radial probability density of target location is proportional to the radius makes it more likely that the target is located, thus detected, closer to the periphery. Incidentally, that is the case in all the panels in Figures 9 and 10, except for Figure 10B, where the target is absent, and Figure $9 \mathrm{C}$, where the target was relatively closer to the center and was correspondingly missed. ${ }^{13}$

Looking for scanpaths, it is just as important to scrutinize the individual behavior as to scrutinize the group behavior. Thus, Keech (2006) compiled tables comparing the proportions of trials with $C<0, C=0$, and $C>0$ for each (young and older) individual participant searching each array size. The results showed no evidence of search patterns consistently adopted by all participants, although some patterns appeared to be favored by certain individuals. Among young adults, for example, Participant 2 showed a slight preference for a clockwise circulation $(C>0)$ for all array sizes, whereas Participants 4 and 5 clearly favored counterclockwise circulation $(C<0)$ for 48- and 96-distractor arrays. Participant 4 also strongly favored a counterclockwise circulation for 12-distractor arrays, but not so for 24-distractor arrays. Participant 5 instead favored clockwise circulation for both those smaller array sizes. Participant 1 behaved similarly to Participant 5, whereas Participants 3, 6, and 7 showed more mixed combinations of "preferences." Among the older adults, Participants 2 and 4 strongly favored clockwise circulation, whereas Participant 6 did the opposite. Participants 1, 3, 5, and 7 showed no clear patterns of "preferences."

Given the randomness in the placement of all the stimuli in our displays, systematic bottom-up stimulation from the scene (besides color segmentation) may derive only from the perception of the display boundary. That might have stimulated some general preference for searching horizontally or vertically, but apparently it did not. The actual distribution of stimuli in each display may have guided to some extent the search of that individual display, but there is no scanning strategy that is generally more efficient than any other for scanning our unstructured collections of two-feature stimuli. So, the individual peculiarities noted by Keech (2006) seem too casual and incidental to suggest scanpaths, each characteristic of a certain individual viewing a certain display, similar in any way to those found instead by Stark and his collaborators in studies of perception and recognition of realistic but marginally visible drawings (Noton \& Stark, 1971; Stark \& Choi, 1996).

Our understanding of the origins of spiraling or circulating eye trajectories (rooted in the three psychoneural mechanisms and the two geometric-spatial constraints that we have already mentioned) permits the development of some crude but insightful estimates that can be compared with various observations. Consider, for example, the case of search trajectories that return to previously inspected or central locations. Although less likely, as already noted, that may still occur for a number of reasons. For example, one may have passed relatively close to the target (on the way out to or around the periphery) but failed to recognize it. Or, in arrays in which the target is absent, the search trajectory may continue until it eventually closes on itself. By crudely modeling the case of a trajectory that spirals out to the periphery and then returns to the center, we may roughly estimate the corresponding number of intervening saccades as follows. The number of saccades executed during a trajectory of a certain length (in degrees) is roughly given by that length divided by the average saccade amplitude, which is about $6.5^{\circ}$ (cf. Figure 7 in Keech et al., 2009). Consider saccading around the periphery of the search array, at a distance of about $2^{\circ}$ from the display boundary, which keeps it within a distance comparable to the $\mathrm{AC}$ radius. Since the dimensions of the screen are $24.4^{\circ} \times 16.3^{\circ}$, the array periphery becomes effectively $2 \cdot\left(22.4^{\circ}+14.3^{\circ}\right) \approx 73^{\circ}$, which can be covered with about 11 saccades. One or 2 additional saccades are typically needed to reach the periphery from the center. Correspondingly, 1 or 2 more saccades are needed to return to the central region, if one has failed to detect the target thus far. For these relatively few occurrences, which may be realized at most for the larger arrays, about 13 to 15 intervening saccades are thus expected. That estimate is consistent with the largest spikes in the normalized rates of return shown in Figures 20C and 20D in Keech et al. (2009), which may thus be regarded as due mainly to returns to previously fixated locations near the center of the display.

We may also use this type of crude estimate to appreciate the saturation effect appearing in the curvilinear relation between area measure and circulation for $|C|>5$. Since about 11 saccades are needed to traverse the screen periphery and 1 or 2 saccades are typically needed to reach that periphery from the center of the screen, about 10 saccades are needed, on average, to first reach and then trace out about three fourths of the screen periphery. That number generally suffices to maximize the change in both the horizontal $(\Delta X)$ and vertical $(\Delta Y)$ displacements, thus maximizing the area measure $A$. Now, 10 saccades comprise a total of $V=9$ angle shifts, or vertices, in between them. Since the average imbalance is about $75 \%$, or $I=3 / 4$, the estimated circulation is about $|C|=V \cdot(2 I-1)=9 / 2$ in magnitude. So, when $C$ exceeds about 5 in magnitude, one should expect that $A$ saturates, meaning that a further increase in $|C|$ ceases to produce any further (curvilinear or linear) increase in $A$. A saturation effect above $|C|=5$ is noticeable in Figures $3 \mathrm{~B}-6 \mathrm{~B}$, which confirms our esti- 
mate. Conversely, we gain further indirect confirmation of the prevalence of spiraling or circulating patterns in the search trajectories, since the saturation estimate depends on that assumption to attain area measure maximization.

\section{DISCUSSION}

We have developed certain statistical analyses and measures of the eye movement trajectories performed during a visual search task in which the color and orientation of the stimuli are the only two separable features that must be integrated into conjunctive wholes in order to identify a target (Keech, 2006; Keech et al., 2009; Motter \& Belky, 1998a, 1998b; Treisman \& Gelade, 1980; Zelinsky \& Sheinberg, 1997). These analyses and measures indicate the following psychoneural and spatial-constraint contributions to the prevalent formation of spiraling or circulating patterns in the eye trajectories.

\section{Autocorrelation Functions: \\ Forward Bias (IOR or Attentional) Contribution}

The statistical analysis of the autocorrelation functions of the saccade direction cosines demonstrates directly the presence of a forward bias in consecutive saccades along the trajectories (Figures 1C and 1D). Such forward bias has been previously noted by other types of analyses (Keech et al., 2009; Motter \& Belky, 1998a; L. G. Williams, 1966, p. 81), and it has been attributed to IOR, so that focal attention can move away from previously fixated locations (Klein \& MacInnes, 1999). An alternative view of IOR and forward bias has also been advanced in terms of attentional momentum, attributing to attention an inertia reminiscent of that of linear momentum in Newtonian mechanics (Spalek \& Hammad, 2004). The cognitive basis of such attentional momentum remains speculative, however, and there are challenges to that view, such as observations of separate object-based and location-based IOR components, possibly resulting from distinct psychoneural mechanisms (Gibson \& Egeth, 1994b; Spalek \& Hammad, 2004, p. 231; Tipper et al., 1994).

\section{Autocorrelation Functions: \\ Geometric and Spatial Constraint Contributions}

The autocorrelation analysis has also demonstrated precisely the effects that two basic geometrical and spatial constraints have on the eye trajectories, which are required to begin near the display center and remain subsequently confined within the rectangular display boundary (see note 9). "Bouncing back" at the display boundary has been shown to counter the forward bias and, in fact, overcome it in the smaller arrays (Figures 1A and 1B), whereas "bouncing around" the screen periphery compatibly with the forward bias contributes with other psychoneural mechanisms to the prevalence of spiraling or circulating eye trajectories, particularly in the larger arrays (as in Figures 9A-9D, for example). Major effects of scene boundaries such as these have not been particularly noted or investigated in other visual search studies, even though most are also conducted with bounded displays or monitors (but see notes 2 and 3).

\section{Power Spectra: Short- and}

\section{Long-Range Memory Contributions}

Both short- and long-range components of "memory" (in the sense specified in note 11) are further revealed by power spectra, which differ from those of white noise at high and low frequencies to various degrees (Figures 2A and $2 \mathrm{~B}$ ), particularly in comparison with those of randomwalk simulations with or without boundaries (Figures B6B and B7B). Short-range "memory" is related to forward bias or IOR with limited capacity, plus the effects of the display boundary in turning saccade directions around (see note 11). Long-range "memory" may be associated with a highcapacity but low-resolution memory for the search path, as described by Dickinson and Zelinsky (2007). Peterson et al. (2001) have also described their finding of a visual search memory for at least 12 items in terms of a grouped representation, or chunking, of items (see also Pashler, 1987). Keech and Resca (in press) have further shown the necessity to include an equivalent form of long-range memory in a computational model (similar to one in Peterson et al., 2001) in order to stand any chance of accounting for the reinspection rates that we observed. Unquestionably, the kind of evidence and understanding of this long-range memory contribution to active visual search is in no way comparable to that of short-range memory or IOR, and much further research will be needed to characterize it anywhere as precisely. Nevertheless, there is at least substantial phenomenological evidence for the existence of such a long-range memory contribution, quite separately or independently of IOR with limited capacity, and our (power spectra and computational) results certainly add to that evidence.

Other authors (Aks, 2005; Aks et al., 2002) have analyzed power spectra of eye fixation series in a task (of finding the upright "T") relatively similar to ours, in order to investigate the occurrence of fractal and $1 / f$ related structures in the dynamics of the oculomotor system. They have concluded, in particular, that long-range memory across eye movements in the form of complex self-organizing search patterns is used to provide maximum coverage of a search area with minimal effort.

\section{Circulation, Imbalance, and Area Measures: Spiraling or Circulating Patterns}

We have then introduced certain measures of circulation $(C)$, imbalance $(I)$, and subtended area $(A)$ that characterize the eye movement trajectories in the conduct of the visual search. Circulating patterns in the eye trajectories are associated with bimodal distributions of $C$ and curvilinear correlations in $A-C$ scatterplots. Such signatures of circulating patterns are unmistakable throughout Figures 3-6, despite their considerable "noise," resulting from the relatively limited number of observations in that regard. By contrast, random-walk trajectories, computationally generated with the same geometrical and spatial constraints, do not exhibit those signatures (there is no trace of bimodality in Figure B5A, e.g.). The prevalence of circulating patterns is even more strikingly demonstrated by the analysis of imbalance in the eye trajectories, where, on average, three out of four saccades typically turn to the same side in a trajectory (detailed characterizations of imbalance are provided 
in the second part of the Circulation, Imbalance, and Area Measures section and in Figures 7 and 8).

\section{Attentional Contribution: The Area of Conspicuity}

The results of these analyses both confirm the original observations of Motter and Belky (1998a, 1998b) and Keech et al. (2009) and depend on their corresponding model, which is summarized in the second paragraph of the introduction to the Our Study section. The AC construct is central to those observations and model. Hence, it is equally essential to accounting for the developments of spiraling or circulating patterns in the eye trajectories. For example, since stimuli are processed in parallel and recognized only within the $\mathrm{AC}$, a good portion of the screen periphery is typically inspected by saccading along the display boundary within an AC radius or so. Since there is also proportionally more area peripherally than centrally, there is a greater chance that the target is located and recognized at a peripheral than at a central location. Thus, there is a prevailing chance that a spiraling trajectory terminates as a circulating pattern near the periphery, before returning randomly toward central locations. Nevertheless, randomly bouncing or closing trajectories are not inherently forbidden and do occur at times. These observations provide the basis for crude but insightful estimates such as those outlined in the last two paragraphs of the Scanning Patterns section, which reasonably explain striking peaks in return rate observations and saturation effects in $A-C$ scatterplots. Thus, our circulation and area measure results and interpretations critically depend on, and fully support, in turn, the characterization of perceptual and attentional elements that have been extensively investigated in previous studies, reviewed briefly in the introduction to the Attentional Resources section.

\section{A Computational Model of Active Visual Search}

The tools and analyses developed in this article demonstrate to a considerable extent the presence of a long-range memory contribution to the visual search task that we investigated. A survivor function analysis of cumulative probabilities of target detection has also indicated that our human participants made considerable use of memory, a result confirmed by the analysis of return rates during the visual search (Keech, 2006; Keech et al., 2009). As has been reported in a companion paper (Keech \& Resca, in press), we have further developed a computational model and corresponding computer simulations that account quantitatively for the same psychoneural mechanisms and geometric-spatial constraints that we found responsible for the pattern formations observed in the eye trajectories. The element of randomness in the visual search is captured through a Monte Carlo selection of a particular eye movement on the basis of its probability, which depends on three factors, adjusted to match the observed saccade amplitude distributions (hence, AC), angle shifts (hence, forward bias), and return rates (hence, long-range memory). These simulations demonstrate that long-range memory, well beyond and in addition to forward bias or IOR with limited memory capacity, is required in order to reproduce all the most critical features of the observations - and return rates, in particular. ${ }^{14}$

On the other hand, our computational model and simulations do not assume or generate any element of predetermination in search trajectories, which is instead typically regarded as characteristic of scanpaths or systematic search strategies, subject to top-down control. Rather, a basic element of randomness persists throughout the entire simulated search trajectory. By applying to the computer-generated trajectories the same analyses (in terms of saccade autocorrelation functions, power spectra, and $C, I$, and $A$ measures) that we have introduced in this article and applied to the observed eye movements, we have shown that essentially the same statistical behaviors can be reproduced for simulated trajectories (Keech \& Resca, in press). In particular, that requires long-range memory to be an essential element of the computational model; otherwise, the observed return rates (as reported in Figures 19 and 20 in Keech et al., 2009) cannot be consistently reproduced computationally for all delays (assuming either no memory or only short-range memory).

\section{Predetermined Scanpaths or Systematic \\ Search Strategies in Unstructured Displays?}

There is ample evidence of use of predetermined scanpaths or systematic search strategies in visual processing of scenes or displays where the presence of any kind of structure may elicit or guide some sort of visual activity particularly compatible with or adaptable to that kind of structure (Findlay \& Brown, 2006a; Gilchrist \& Harvey, 2006; Noton \& Stark, 1971; Peterson et al., 2007; Peterson et al., 2002; Stark \& Choi, 1996; Yarbus, 1967). In our introduction to the Search Strategies section, we have briefly reviewed some of that evidence and literature.

Not surprisingly, the visual system must be quite adept and finely tuned to perceive any structure or regularity in a scene that may guide or help the visual search if it adopts some correspondingly suited or adaptable scanning strategy or scanpath. The outstanding question, at least from our perspective, is whether or to what extent there can remain a persistence of physiological, phylogenetic, or idiosyncratic viewing biases characteristic of the observer when that person or animal is presented with a completely unstructured and randomized distribution of stimuli, for which there is no objective advantage of any one scanning strategy over any other.

For example, Lévy-Schoen (1981) has suggested physiological preferences for horizontal and vertical oculomotor scanning and other possible constraints on ocular motility. Cohen and Ruppin (1999) have noted that "vertical symmetry, a fundamental characteristic of natural scenes, plays a central role in visual-search tasks. This is demonstrated in Wolfe's work (Wolfe, Friedman-Hill, Stewart, \& O'Connell, 1992)" (p. 1457). In that regard, we may further contemplate that life is indeed embedded in an environment that is inherently biased vertically and horizontally by gravity. The horizon (where an animal may search for prey or predators) is laid out horizontally. Trees (where an animal may look for fruits or branches) are vertical. Different cultures have developed different layouts 
for writing and reading, but most are either horizontal or vertical. So, it is certainly conceivable that there is at least some horizontal or vertical bias inherent to the oculomotor or the visuospatial attentional system (as suggested also by Spalek \& Hammad, 2004). The best, if not the only, way to find out is to use completely unstructured and randomized distributions of stimuli, as we did, although further study of possible effects of the display boundary should include changes and rotations of the shape and size of that boundary, for example.

So, what have we found so far? According to our combined analyses of saccade autocorrelations and $C, I$, and $A$ measures, there is definitely a prevalence of spiraling or circulating patterns in the eye movement trajectories. Beyond that, we looked for but found no clear evidence of other scanning patterns, such as scanning left to right, top to bottom, rasterlike, snakelike, hub and spokes, and so forth. Now, what is the basic origin and nature of the spiraling or circulating patterns that we found in our unstructured and randomized distributions of stimuli? Our computational model indicates that predetermination is not required for those circulating patterns to develop. On the other hand, random-walk simulations indicate that geometric and spatial constraints alone generate no prevalence of circulating patterns (e.g., there is no trace of bimodality in the circulation $C$ distribution in Figure B5A). Thus, some psychoneural mechanisms must further adapt and combine with the geometrical and spatial constraints of the task to generate the observed prevalence of circulating patterns in eye trajectories. We identified those psychoneural mechanisms as related to the $\mathrm{AC}$, forward bias in consecutive saccade directions, and long-range memory (e.g., as summarized in the last paragraph of the introduction to the Our Study section).

However, other conceptualizations or interpretations of our findings are certainly possible and should never be categorically excluded. In fact, nothing that we found actually forbids regarding a circulating pattern as the possible result of some predetermined search strategy or scanpath, whether deliberately intended or automatically preprogrammed as a consequence of experience or innate bias. For example, some individual participants in our experiments predominantly selected either clockwise or counterclockwise circulating search patterns, at least for some array sizes (see the Scanning Patterns section and Keech, 2006, for further details). On the other hand, there does not seem to be any consistency for such attitudes or biases between various individuals or even within the same individual for various array sizes. What to make of all that? We are personally inclined to think that such or other, similar individual peculiarities are too casual and incidental to provide convincing evidence of consistent predetermination of scanning in the visual search of completely unstructured and randomized distributions of stimuli, at least for a typical, or "average," individual observer. But other researchers may find additional or alternative evidence to convince them otherwise, and in fact, we encourage them to do so.

To give another example, we certainly would have subjected our data to the same analysis that generated
Figure 2 in the article by Gilchrist and Harvey (2006), in order to test whether systematic strategies, such as rasterscanning, might persist in our unstructured displays, as they do in their grid-structured displays. We suppose that they would not, but we have lost all our data and can no longer pursue this further interesting investigation. Conversely, if Gilchrist and Harvey (2006) had known of our techniques and measures, they could have applied them to their data and, presumably, obtained an entire set of results completely different from ours. For example, they could have found unimodal $C$ distributions, narrowly peaked at $C=0$, with little or no imbalance, which would be consistent with raster- or reading-like scanning, as opposed to prevailing circulation to either side.

\section{Boundless Scenes}

Another line of investigation that we conceived of but can no longer pursue is the following. On the basis of our analyses and conclusions, removal of geometric-spatial constraints peculiar to the task could reduce or eliminate the prevalence of circulating trajectories and their corresponding $C, I$, and $C-A$ signatures. This could be tested in part with experiments in which participants are required initially to fixate a cross that appears randomly anywhere in the display, rather than systematically at its center. Further removal of the boundary constraint may be achieved by viewing a virtual reality display corresponding to a boundless scene. That should eliminate any residual prevalence of circulating trajectories, since those patterns are presumably not stereotypically inherent to the oculomotor or the visuospatial attentional system but, rather, are the result of the interaction of psychoneural mechanisms with geometric-spatial constraints specific to the prototypical task that we investigated.

\section{CONCLUSIONS}

So, with specific regard to both memory and scanning patterns, the aggregate of our results and conclusions is most consistent with previous results and conclusions in Dickinson and Zelinsky (2005) and Peterson et al. (2001). That is probably not coincidental, since their displays were both stable and unstructured, just like ours. Accordingly, we support their more or less equivalent concepts of a high-capacity but low-resolution memory for the search path (Dickinson \& Zelinsky, 2005), or a grouped/chunked representation of it (Peterson et al., 2001). That search path, however, may be one that has occurred for whatever reason, like spiraling or circulating trajectories that prevailed in, and partly because of, our specific task and displays, and not necessarily one that is either predetermined as a scanpath or the result of some more systematic search strategy. Those are more likely to be perceptually elicited in at least partly structured displays, like those in Gilchrist and Harvey (2006) or Peterson et al. (2007), resulting more likely in prospective memory.

In closing, we would also like to mention that, although it took us a long time (since at least 2002) to collect our data and analyze them with our techniques, the autocorrelation functions, power spectra, and the circulation, im- 
balance, and area measures that we ultimately developed depend entirely on fairly elementary mathematics and can be computed directly with relatively straightforward and unsophisticated programming. Thus, it would be quite feasible and interesting (we think) for some researchers (such as Aks, Motter, Peterson, and others) who already have comparable or even more extensive sets of visual search data to analyze them with the same techniques or ones similar to ours and, thus, determine the extent to which the eye movement characteristics and patterns that we have identified may be relatively common to relatively similar visual search tasks (as we expect).

\section{AUTHOR NOTE}

The authors are greatly indebted to Pamela Greenwood and Raja Parasuraman for introducing them to and training them in this research area and project. The authors are also greatly indebted to Brad Motter for countless and extremely valuable comments and suggestions throughout this work. L.R. is grateful to Bradley Gibson, Todd Horowitz, and Matthew Peterson for their thorough reviews and critical contributions to both the substance and presentation of the manuscript. The final version of this article reflects and depends in a large measure on a great deal of assistance and expertise provided generously by each of the individuals mentioned above. Technical assistance by Richard Cecil is also gratefully acknowledged. It is with great sorrow that L.R. acknowledges the loss of T.D.K., his former student and dear colleague. Correspondence concerning this article should be addressed to L. Resca, Physics Department, Catholic University of America, Washington, DC 20064 (e-mail: resca@cua.edu).

\section{REFERENCES}

AKs, D. J. (2005). 1/ $f$ dynamic in complex visual search: Evidence for self-organized criticality in human perception. In M. A. Riley \& G. C. Van Orden (Eds.), Tutorials in contemporary nonlinear methods for the behavioral sciences (pp. 326-359). Arlington, VA: National Science Foundation. Available at www.nsf.gov/sbe/bcs/pac/nmbs/nmbs .jsp.

AKs, D. J., Zelinsky, G., \& Sprott, J. C. (2002). Memory across eyemovements: $1 / f$ dynamic in visual search. Nonlinear Dynamics, Psychology, \& Life Sciences, 6, 1-25.

Ball, K. K., Beard, B. L., Roenker, D. L., Miller, R. L., \& Griggs, D. S. (1988). Age and visual search: Expanding the useful field of view. Journal of the Optical Society of America, 5, 2210-2219.

BAYLIS, G. C., \& DrIVER, J. (1993). Visual attention and objects: Evidence for hierarchical coding of location. Journal of Experimental Psychology: Human Perception \& Performance, 19, 451-470.

Beck, M. R., Peterson, M. S., Boot, W. R., Vomela, M., \& Kramer, A. F. (2006). Explicit memory for rejected distractors during visual search. Visual Cognition, 14, 150-174.

Beck, M. R., Peterson, M. S., \& Vomela, M. (2006). Memory for where, but not what, is used during visual search. Journal of Experimental Psychology: Human Perception \& Performance, 32, 235-250.

Boccignone, G., \& Ferraro, M. (2004). Modelling gaze shifts as a constrained random walk. Physica A, 331, 207-218.

Boot, W. R., McCarley, J. S., Kramer, A. F., \& Peterson, M. S. (2004). Automatic and intentional memory processes in visual search. Psychonomic Bulletin \& Review, 11, 854-861.

Brockmann, D., \& Geisel, T. (2000). The ecology of gaze shifts. Neurocomputing, 32, 643-650.

Cameron, E. L., Tai, J. C., Eckstein, M. P., \& Carrasco, M. (2004). Signal detection theory applied to three visual search tasksIdentification, yes/no detection and localization. Spatial Vision, 17, 295-325.

Castiello, U., \& Umiltà, C. (1990). Size of the attentional focus and efficiency of processing. Acta Psychologica, 73, 195-209.

CAVE, K. R., \& Bichot, N. P. (1999). Visuospatial attention: Beyond a spotlight model. Psychonomic Bulletin \& Review, 6, 204-223.

CAVE, K. R., \& Wolfe, J. M. (1990). Modeling the role of parallel processing in visual search. Cognitive Psychology, 22, 225-271.
Chastain, G. (1992). Analog versus discrete shifts of attention across the visual field. Psychological Research, 54, 175-181.

Cohen, E., \& Ruppin, E. (1999). From parallel to serial processing: A computational study of visual search. Perception \& Psychophysics, 61, 1449-1461.

Danziger, S., Kingstone, A., \& Snyder, J. J. (1998). Inhibition of return to successively stimulated locations in a sequential visual search paradigm. Journal of Experimental Psychology: Human Perception \& Performance, 24, 1467-1475.

Deubel, H., \& Schneider, W. X. (1996). Saccade target selection and object recognition: Evidence for a common attentional mechanism. Vision Research, 36, 1827-1837.

Dickinson, C. A., \& Zelinsky, G. J. (2005). Marking rejected distractors: A gaze-contingent technique for measuring memory during search. Psychonomic Bulletin \& Review, 12, 1120-1126.

Dickinson, C. A., \& ZeLINSKY, G. J. (2007). Memory for the search path: Evidence for a high-capacity representation of search history. Vision Research, 47, 1745-1755.

Dodd, M. D., Castel, A. D., \& Pratt, J. (2003). Inhibition of return with rapid serial shifts of attention: Implications for memory and visual search. Perception \& Psychophysics, 65, 1126-1135.

DownING, C. J. (1988). Expectancy and visual-spatial attention: Effects on perceptual quality. Journal of Experimental Psychology: Human Perception \& Performance, 14, 188-202.

Duncan, J. (1984). Selective attention and the organization of visual information. Journal of Experimental Psychology: General, 113, 501-517.

Duncan, J., \& Humphreys, G. W. (1989). Visual search and stimulus similarity. Psychological Review, 96, 433-458.

DunCAN, J., \& HumphreYs, G. W. (1992). Beyond the search surface: Visual search and attentional engagement. Journal of Experimental Psychology: Human Perception \& Performance, 18, 578-588.

Eckstein, M. P., Thomas, J. P., Palmer, J., \& Shimozaki, S. S. (2000). A signal detection model predicts the effects of set size on visual search accuracy for feature, conjunction, triple conjunction, and disjunction displays. Perception \& Psychophysics, 62, 425-451.

EgETH, H. E. (1977). Attention and preattention. In G. H. Bower (Ed.), The psychology of learning and motivation (Vol. 11, pp. 277-320) New York: Academic Press.

Egeth, H. E., Virzi, R. A., \& Garbart, H. (1984). Searching for conjunctively defined targets. Journal of Experimental Psychology: Human Perception \& Performance, 10, 32-39.

ENGEL, F. L. (1971). Visual conspicuity, directed attention and retinal locus. Vision Research, 11, 563-576.

ENGEL, F. L. (1974). Visual conspicuity and selective background interference in eccentric vision. Vision Research, 14, 459-471.

ENGEL, F. L. (1977). Visual conspicuity, visual search and fixation tendencies of the eye. Vision Research, 17, 95-108.

ERIKSEN, C. W., \& Murphy, T. D. (1987). Movement of the attentional focus across the visual field: A critical look at the evidence. Perception \& Psychophysics, 42, 299-305.

ERIKSEN, C. W., \& St. JAMES, J. D. (1986). Visual attention within and around the field of focal attention: A zoom lens model. Perception \& Psychophysics, 40, 225-240.

FINDLAY, J. M. (1997). Saccade target selection during visual search. $\mathrm{Vi}$ sion Research, 37, 617-631.

Findlay, J. M., \& Brown, V. (2006a). Eye scanning of multi-element displays: I. Scanpath planning. Vision Research, 46, 179-195.

Findlay, J. M., \& BROWN, V. (2006b). Eye scanning of multi-element displays: II. Saccade planning. Vision Research, 46, 216-227.

Findlay, J. M., Brown, V., \& Gilchrist, I. D. (2001). Saccade target selection in visual search: The effect of information from the previous fixation. Vision Research, 41, 87-95.

FindLAY, J. M., \& WaLKeR, R. (1999). A model of saccadic eye movement generation based on parallel processing and competitive inhibition. Behavioral \& Brain Sciences, 22, 661-721.

Gibson, B. S. (1994). Visual attention and objects: One versus two or convex versus concave? Journal of Experimental Psychology: Human Perception \& Performance, 20, 203-207.

Gibson, B. S., \& EgETh, H. (1994a). Inhibition and disinhibition of return: Evidence for temporal order judgments. Perception \& Psychophysics, 56, 669-680.

GibSON, B. S., \& EGETH, H. (1994b). Inhibition of return to object-based 
and environment-based locations. Perception \& Psychophysics, 55, 323-339.

Gibson, B. S., Li, L., Skow, E., Brown, K., \& Cooke, L. (2000). Searching for one versus two identical targets: When visual search has memory. Psychological Science, 11, 324-327.

Gilchrist, I. D., \& Harvey, M. (2000). Refixation frequency and memory mechanisms in visual search. Current Biology, 10, 1209-1212.

Gilchrist, I. D., \& HaRveY, M. (2006). Evidence for a systematic component within scan paths in visual search. Visual Cognition, 14, 704-715.

Gilchrist, I. D., NorTh, A., \& HooD, B. (2001). Is visual search really like foraging? Perception, 30, 1459-1464.

Green, D. M., \& Swets, J. A. (1966). Signal detection theory and psychophysics. New York: Krieger.

Greenwood, P. M., \& Parasuraman, R. (1999). Scale of attentional focus in visual search. Perception \& Psychophysics, 61, 837-859.

Greenwood, P. M., \& Parasuraman, R. (2004). The scaling of spatial attention in visual search and its modification in healthy aging. Perception \& Psychophysics, 66, 3-22.

HENDERSON, J. M. (1992). Visual attention and eye-movement control during reading and picture viewing. In K. Rayner (Ed.), Eye movements and visual cognition: Scene perception and reading (pp. 260283). New York: Springer.

HENDERSON, J. M. (1993). Visual attention and saccadic eye movements. In G. d'Ydewalle \& J. van Rensbergen (Eds.), Perception and cognition: Advances in eye movement research (pp. 37-50). Amsterdam: Elsevier.

Hooge, I. T. C., \& Erkelens, C. J. (1996). Control of fixation duration in a simple search task. Perception \& Psychophysics, 58, 969-976.

Hooge, I. T. C., \& ERKELENS, C. J. (1998). Adjustment of fixation duration in visual search. Vision Research, 38, 1295-1302.

Hooge, I. T. C., \& ERKelens, C. J. (1999). Peripheral vision and oculomotor control during visual search. Vision Research, 39, 1567-1575.

Horowitz, T. S., \& Wolfe, J. M. (1998). Visual search has no memory. Nature, 394, 575-577.

Horowitz, T. S., \& Wolfe, J. M. (2001). Search for multiple targets: Remember the targets, forget the search. Perception \& Psychophysics, 63, 272-285.

Horowitz, T. S., \& Wolfe, J. M. (2003). Memory for rejected distractors in visual search? Visual Cognition, 10, 257-298.

IKEDA, M., \& TAKEUCHI, T. (1975). Influence of foveal load on the functional visual field. Perception \& Psychophysics, 18, 255-260.

Irwin, D. E., \& ZelinsKy, G. J. (2002). Eye movements and scene perception: Memory for things observed. Perception \& Psychophysics, 64, 882-895.

IтTI, L., \& Косн, C. (2000). A saliency-based search mechanism for overt and covert shifts of visual attention. Vision Research, 40, 1489-1506.

Iтti, L., \& Kосн, C. (2001). Computational modeling of visual attention. Nature Reviews Neuroscience, 2, 1-11.

JuLESZ, B. (1981). A theory of preattentive texture discrimination based on first order statistics of textons. Biological Cybernetics, 41, 131-138.

JuLESZ, B. (1984). A brief outline of the texton theory of human vision. Trends in Neurosciences, 7, 41-45.

Julesz, B., \& Bergen, J. R. (1983). Textons, the fundamental elements in preattentive vision and perception of textures. Bell Systems Technology, 62, 1619-1646.

KeEch, T. D. (2006). Dynamics of spontaneous saccades in a conjunctive visual search task. Doctoral thesis, Catholic University of America, Department of Physics. Full-text PDF available from http:// libraries.cua.edu/welcome.html under the Dissertations from CUA database selection.

Keech, T. D., Greenwood, P. M., Resca, L., \& Parasuraman, R. (2009). Active visual search in young and older humans: The interplay of attention and memory. Unpublished manuscript.

KeECH, T. D., \& ReSCA, L. (in press). Eye movements in active visual search: A computable phenomenological model. Attention, Perception, \& Psychophysics.

KLEIN, R. [M.] (1988). Inhibitory tagging system facilitates visual search. Nature, 334, 430-431.

KLEIN, R. M. (2000). Inhibition of return. Trends in Cognitive Sciences, 4, 138-147.

KLEIN, R. M., \& MACINNES, W. J. (1999). Inhibition of return is a foraging facilitator in visual search. Psychological Science, 10, 346-352.
KleIN, R. M., \& TAYLOR, T. L. (1994). Categories of cognitive inhibition with reference to attention. In D. Dagenbach \& T. H. Carr (Eds.), Inhibitory processes in attention, memory, and language (pp. 113-150). San Diego: Academic Press.

Koch, C., \& Ullman, S. (1985). Shifts in selective visual attention: Toward the underlying neural circuitry. Human Neurobiology, 4, 219-227.

Kowler, E., Anderson, E., Dosher, B., \& Blaser, E. (1995). The role of attention in the programming of saccades. Vision Research, 35, 1897-1916.

Kraiss, K. F., \& KnäEuper, A. (1982). Using visual lobe area measurements to predict visual search performance. Human Factors, 24, 673-682.

Kristjánsson, Á. (2000). In search of remembrance: Evidence for memory in visual search. Psychological Science, 11, 328-332.

LaBerge, D., \& Brown, V. (1989). Theory of attentional operations in shape identification. Psychological Review, 96, 101-124.

LÉVY-SCHOEN, A. (1981). Flexible and/or rigid control of oculomotor scanning behavior. In D. Fisher, R. A. Monty, \& J. Sender (Eds.), Eye movements: Cognition and visual perception (pp. 299-314). Hillsdale, NJ: Erlbaum.

Loftus, G. R., \& Masson, M. E. J. (1994). Using confidence intervals in within-subject designs. Psychonomic Bulletin \& Review, 1, 476-490.

Lupiáñez, J., Klein, R. M., \& Bartolomeo, P. (2006). Inhibition of return: Twenty years after. Cognitive Neuropsychology, 23, 1003-1014.

Masson, M. E. J., \& Loftus, G. R. (2003). Using confidence intervals for graphically based data interpretation. Canadian Journal of Experimental Psychology, 57, 203-220.

McCarley, J. S., Wang, R. F., Kramer, A. F., Irwin, D. E., \& Peterson, M. S. (2003). How much memory does oculomotor search have? Psychological Science, 14, 422-426.

McPeek, R. M., Skavenski, A. A., \& Nakayama, K. (2000). Concurrent processing of saccades in visual search. Vision Research, 40, 2499-2516.

Motter, B. C., \& Belky, E. J. (1998a). The guidance of eye movements during active visual search. Vision Research, 38, 1805-1815.

Motter, B. C., \& BeLky, E. J. (1998b). The zone of focal attention during active visual search. Vision Research, 38, 1007-1022.

Motter, B. C., \& Holsapple, J. W. (2000). Cortical image density determines the probability of target discovery during active search. Vision Research, 40, 1311-1322.

Motter, B. C., \& Holsapple, J. W. (2001). Separating attention from chance in active visual search. In J. Braun, C. Koch, \& J. Davis (Eds.), Visual attention and neural circuits (pp. 159-175). Cambridge, MA: MIT Press.

Motter, B. C., \& Holsapple, J. W. (2007). Saccades and covert shifts of attention during active visual search: Spatial distributions, memory, and items per fixation. Vision Research, 47, 1261-1281.

Motter, B. C., \& Simoni, D. A. (2007). The roles of cortical image separation and size in active visual search performance. Journal of $V i$ sion, 7(2, Art. 6), 1-15. Available at http://journalofvision.org/7/2/6/. doi: $10.1167 / 7.2 .6$

Motter, B. C., \& Simoni, D. A. (2008). Changes in the functional visual field during search with and without eye movements. Vision Research, 48, 2382-2393.

MülleR, H. J., \& von MüHLEnEN, A. (2000). Probing distractor inhibition in visual search: Inhibition of return. Journal of Experimental Psychology: Human Perception \& Performance, 32, 1591-1605.

Müller, N. G., Bartelt, O. A., Donner, T. H., Villringer, A., \& BRANDT, S. A. (2003). A physiological correlate of the "zoom lens" of visual attention. Journal of Neuroscience, 23, 3561-3565.

Murphy, T. D., \& ERIKsen, C. W. (1987). Temporal changes in the distribution of attention in the visual field in response to precues. Perception \& Psychophysics, 42, 576-586.

NajemniK, J., \& Geisler, W. S. (2005). Optimal eye movement strategies in visual search. Nature, 434, 387-391.

Noton, D., \& Stark, L. W. (1971). Scanpaths in eye movements during pattern perception. Science, 171, 308-311.

OpPenheim, A. V., SCHAFer, R. W., \& BucK, J. R. (1999). Discrete-time signal processing (2nd ed.). Englewood Cliffs, NJ: Prentice Hall.

Palmer, J., Verghese, P., \& Pavel, M. (2000). The psychophysics of visual search. Vision Research, 40, 1227-1268. 
PAshler, H. (1987). Detecting conjunctions of color and form: Reassessing the serial search hypothesis. Perception \& Psychophysics, 41, 191-201

Pathria, R. K. (1996). Statistical mechanics (2nd ed.). Oxford: Butterworth-Heinemann.

Peterson, M. S., Beck, M. R., \& Vomela, M. (2007). Visual search is guided by prospective and retrospective memory. Perception \& Psychophysics, 69, 123-135.

Peterson, M. S., Kramer, A. F., \& Irwin, D. E. (2004). Covert shifts of attention precede involuntary eye movements. Perception \& Psychophysics, 66, 398-405.

Peterson, M. S., Kramer, A. F., Irwin, D. E., \& Hahn, S. (2002). Modulation of oculomotor capture by abrupt onsets during attentionally demanding visual search. Visual Cognition, 9, 755-791.

Peterson, M. S., Kramer, A. F., Wang, R. F., Irwin, D. E., \& McCarley, J. S. (2001). Visual search has memory. Psychological Science, 12, 287-292.

Pomplun, M., Reingold, E. M., \& Shen, J. (2003). Area activation: A computational model of saccadic selectivity in visual search. Cognitive Science, 27, 299-312.

Posner, M. I., \& Cohen, V. (1984). Components of visual orienting. In H. Bouma \& D. G. Bouwhuis (Eds.), Attention and performance X: Control of language processes (pp. 531-556). Hillsdale, NJ: Erlbaum.

Posner, M. I., Rafal, R. D., Choate, L. S., \& Vaughan, J. (1985) Inhibition of return: Neural basis and function. Cognitive Neuropsychology, 2, 211-228.

SAARINEN, J., \& JulEsz, B. (1991). The speed of attentional shifts in the visual field. Proceedings of the National Academy of Sciences, 88, 1812-1814.

SAGI, D., \& Julesz, B. (1985). Fast noninertial shifts of attention. Spatial Vision, 1, 141-149.

SANDERS, A. F. (1970). Some aspects of the selective process in the functional visual field. Ergonomics, 13, 101-117.

Sanders, A. F., \& Houtmans, M. J. M. (1985). Perceptual processing modes in the functional visual field. Acta Psychologica, 58, 251-261.

Scialfa, C. T., Kline, D. W., \& Lyman, B. J. (1987). Age differences in target identification as a function of retinal location and noise level: An examination of the useful field of view. Psychological Aging, 2, 14-19.

Scialfa, C. T., Thomas, D. M., \& Joffe, K. M. (1994). Age differences in the useful field of view: An eye movement analysis. Optometry \& Vision Science, 71, 736-742.

Scinto, L. F. M., Pillalamarri, R., \& Karsh, R. (1986). Cognitive strategies for visual search. Acta Psychologica, 62, 263-292.

Shore, D. I., \& KLEIN, R. M. (2000). On the manifestations of memory in visual search. Spatial Vision, 14, 59-75.

SNyder, J. J., \& KingSTONE, A. (2000). Inhibition of return and visual search: How many separate loci are inhibited? Perception \& Psychophysics, 62, 452-458.

SpaleK, T. M., \& Hammad, S. (2004). Supporting the attentional momentum view of IOR: Is attention biased to go right? Perception \& Psychophysics, 66, 219-233.

Sperling, G., \& Weichselgartner, E. (1995). Episodic theory of the dynamics of spatial attention. Psychological Review, 102, 503-532.

StARK, L. W., \& ChoI, Y. S. (1996). Experimental metaphysics: The scanpath as an epistemological mechanism. In W. H. Zangemeister, H. S. Stiehl, \& C. Freksa (Eds.), Visual attention and cognition (pp. 3-69). Amsterdam: Elsevier.

Swets, J. A. (ED.) (1964). Signal detection and recognition by human observers: Contemporary readings. New York: Wiley.

TAKEDA, Y. (2004). Search for multiple targets: Evidence for memorybased control of attention. Psychonomic Bulletin \& Review, 11, 71-76.

TAKEDA, Y., \& YAGI, A. (2000). Inhibitory tagging in visual search can be found if search stimuli remain visible. Perception \& Psychophysics, 62, 927-934.

TANNER, W. P., JR., \& Swets, J. A. (1954). A decision-making theory of visual detection. Psychological Review, 61, 401-409.

TAYlOR, T. L., \& KlEIN, R. M. (1998). On the causes and effects of inhibition of return. Psychonomic Bulletin \& Review, 5, 625-643.

Thornton, I. M., \& Horowitz, T. S. (2004). The multi-item localization (MILO) task: Measuring the spatiotemporal context of vision for action. Perception \& Psychophysics, 66, 38-50.

Tipper, S. P., Weaver, B., Jerreat, L. M., \& Burak, A. L. (1994). Object-based and environment-based inhibition of return of visual attention. Journal of Experimental Psychology: Human Perception \& Performance, 20, 478-499.

Torralba, A., Oliva, A., Castelhano, M. S., \& Henderson, J. M. (2006). Contextual guidance of eye movements and attention in realworld scenes: The role of global features on object search. Psychological Review, 113, 766-786.

Treisman, A. [M.] (1988). Features and objects: The 14th Bartlett Memorial Lecture. Quarterly Journal of Experimental Psychology, 40A 201-237.

Treisman, A. M., \& Gelade, G. (1980). A feature-integration theory of attention. Cognitive Psychology, 12, 97-136.

Treisman, A. [M.], \& Gormican, S. (1988). Feature analysis in early vision: Evidence from search asymmetries. Psychological Review, 95, $15-48$

Treisman, A. [M.], \& Sato, S. (1990). Conjunction search revisited. Journal of Experimental Psychology: Human Perception \& Performance, 16, 459-478.

Unema, P. J. A., Pannasch, S., Joos, M., \& Velichkovsky, B. M. (2005). Time course of information processing during scene perception: The relationship between saccade amplitude and fixation duration. Visual Cognition, 12, 473-494

Verghese, P. (2001). Visual search and attention: A signal detection theory approach. Neuron, 31, 523-535.

von Mühlenen, A., Müller, H. J., \& Müller, D. (2003). Sit-andwait strategies in dynamic visual search. Psychological Science, 14, 309-314

WidDEL, H. (1983). A method of measuring the visual lobe area. In R. Groner, C. Menz, D. F. Fisher, \& R. A. Monty (Eds.), Eye movements and psychological functions: International views (pp. 73-83). Hillsdale, NJ: Erlbaum.

Widdel, H., \& Kaster, J. (1981). Eye movement measurements in the assessment and training of visual performance. In J. Moraal \& K. F. Kraiss (Eds.), Manned systems design, methods, equipment and applications (pp. 251-270). New York: Plenum.

Williams, D. E., \& Reingold, E. M. (2001). Preattentive guidance of eye movements during triple conjunction search tasks: The effects of feature discriminability and saccadic amplitude. Psychonomic Bulletin \& Review, 8, 476-488.

Williams, L. G. (1966). Target conspicuity and visual search. Human Factors, 8, 80-92.

Wolfe, J. M. (1994). Guided Search 2.0: A revised model of visual search. Psychonomic Bulletin \& Review, 1, 202-238.

Wolfe, J. M. (1998). Visual search. In H. Pashler (Ed.), Attention (pp. 13-73). Hove, U.K.: Psychology Press.

WoLfE, J. M. (2003). Moving toward solutions to some enduring controversies in visual search. Trends in Cognitive Sciences, 7, 70-76.

Wolfe, J. M., CaVe, K. R., \& Franzel, S. L. (1989). Guided search: An alternative to the feature integration model for visual search. Journal of Experimental Psychology: Human Perception \& Performance, 15, 419-433.

Wolfe, J. M., Friedman-Hill, S. R., Stewart, M. I., \& O'Connell, K. M. (1992). The role of categorization in visual search for orientation. Journal of Experimental Psychology: Human Perception \& Performance, 18, 34-49.

Wolfe, J. M., \& PoKorny, C. W. (1990). Inhibitory tagging in visual search: A failure to replicate. Perception \& Psychophysics, 48, $357-$ 362

Woodman, G. F., \& LUCK, S. J. (2004). Visual search is slowed when visuospatial working memory is occupied. Psychonomic Bulletin \& Review, 11, 269-274.

Woodman, G. F., Vogel, E. K., \& Luck, S. J. (2001). Visual search remains efficient when visual working memory is full. Psychological Science, 12, 219-224.

YARbus, A. L. (1967). Eye movements and vision (L. A. Riggs, Trans.). New York: Plenum.

ZELINSKY, G. J. (1996). Using eye saccades to assess the selectivity of search movements. Vision Research, 36, 2177-2187.

Zelinsky, G. J., \& LoschKy, L. C. (2005). Eye movements serialize memory for objects in scenes. Perception \& Psychophysics, 67, 676-690.

Zelinsky, G. J., Rao, R. P. N., Hayhoe, M. M., \& Ballard, D. H. (1997). Eye movements reveal the spatiotemporal dynamics of visual search. Psychological Science, 8, 448-453.

Zelinsky, G. J., \& Sheinberg, D. L. (1997). Eye movements during 
parallel-serial visual search. Journal of Experimental Psychology: Human Perception \& Performance, 23, 244-262.

ZiHL, J., \& HeBEL, N. (1997). Patterns of oculomotor scanning in patients with unilateral posterior parietal or frontal lobe damage. Neuropsychologia, 35, 893-906.

\section{NOTES}

1. For alternative views of object-based attention, see also Duncan (1984), Baylis and Driver (1993), and Gibson (1994).

2. For an interesting contextual guidance model of selective perception, combining low-level features with global scene context, see Torralba, Oliva, Castelhano, and Henderson (2006) and related references.

3. Gilchrist and Harvey (2006) have similarly reported 3.3\%, 4.5\%, and $6.1 \%$ of fixations outside the display area in the three conditions in their experiments.

4. Target-absent versus target-present slope ratios greater than 2:1 are more typically observed (Wolfe, 1998; Zelinsky \& Sheinberg, 1997), but slope ratios closer to 1:1 also occur for displays with sparser items (Pashler, 1987).

5. See, for example, in Figures B5 and B8, the $A-C$ characteristics of random-walk simulations, even though those simulations assume that all saccades have equal amplitudes and all fixations have equal durations.

6. The 9:1 target-present/absent ratio was reasonably chosen to discourage participants from prematurely guessing or falsely reporting the presence of targets, while still collecting a greater number of targetpresent trials, since only those could be used later for comparison with computer simulations (Keech, 2006; Keech \& Resca, in press).

7. Our older participants were apparently not "old" enough to generate statistically significant differences between their age group and that of our "young" participants on most measures. Nevertheless, various trends in those differences were suggestive and worth considering, which is why data were kept and analyzed separately and completely for the two groups in our original work (Keech, 2006; Keech et al., 2009). Here, we are not interested in age-related differences, since they do not affect in any meaningful or significant way any of the results or conclusions that we report or reach in this article. Because of space limitations, we often report here data or results for just one age group, while mentioning the corresponding results for the other age group (see Figures 7 and 8 for an exception). We often choose the group of older adults, for no particular reason. Data for the other age group, or the two groups combined, could have been reported equivalently, without any meaningful consequence for this article. Complete sets of data and results for both age groups are instead provided in Keech's (2006) doctoral thesis, accessible on the Internet.

8. Later on, we may also compare Figures B6A and B7A for randomwalk simulations with and without a rectangular boundary constraint. In those simulations, all psychoneural mechanisms are removed, and the one and only mechanism that can and does turn $R(l)=0$ for $l>0$ in Figure B7A into $R(l)<0$ for $l>0$ in Figure B6A is precisely the boundary constraint.

9. We should point out that one might picture saccadic eye movements within the display as those of balls bouncing at the edges of a billiard or some other sort of "mechanical" apparatus. Such figurative language or imagery may be harmless or even helpful at times, but it clearly has no physical basis and obvious limitations. The display boundary operates not as a physical but, rather, as a psychic boundary to the eye movements. Saccades tend to target search items, and the oculomotor system perceives that there are none of those outside the display, thus preventing saccades from straying out of the display boundary, for the most part. Now, the process of statistical averaging in the autocorrelation function $R(l)$ is designed to eliminate any random component in the saccadic eye movement directions and, thus, let survive and demonstrate only their systematic components. In our application, those systematic components are only the tendencies of the saccade directions to progress intrinsically forward, but without crossing the display perimeter-hence, turning backward or "around" therein, figuratively speaking.
10. For the older adults, the average number of saccades and the corresponding standard deviation in 12-distractor arrays are 3.3 and 2.0, respectively, whereas in 96-distractor arrays they are 8.4 and 4.6, respectively. See Figures 2 and 3 and Section 3.1 in Keech et al. (2009) for a presentation and discussion of saccade number distributions in our visual search task.

11. Recall from the descriptions provided just before and after Equations 1-3 that what counts as "memory" in an autocorrelation function (hence, its power spectrum) is any systematic component that overcomes randomness in the sequential values attained by the fluctuating variable. The display boundary disrupts randomness in the direction of saccades by forcing them to turn around when they reach it. Since that disruption occurs fairly soon after the initial central fixation, we expect the boundary constraint to contribute substantially to the short-range "memory" components in saccade autocorrelations and corresponding power spectra. Now, a bias to progress forward in saccade direction also overcomes randomness in saccade direction generation, inherently. Since that affects consecutive saccades, its "memory" contribution is also short-range.

Furthermore, we should point out that the forward bias may be related to IOR or attentional momentum (see the Discussion section) or some (other) form of top-down control. Whatever the theoretical description, we may regard forward bias as a psychoneural mechanism, intrinsically. On the other hand, the display boundary acts as a spatial constraint on the eye movement trajectories, although that action must also derive from a psychoneural mechanism, ultimately (cf. note 9). Regardless of their rather different endogenous or exogenous psychoneural origins, the forward bias and the boundary constraint contribute together, mathematically, to short-range "memory" components in the power spectral analysis of the saccade direction cosine autocorrelation, although for opposite reasons: concurring bias in consecutive saccades versus prompt "reflections" at the display boundary. To avoid possible confusions or unwarranted implications, we encourage each reader to always reflect on the meaning of terms that we use in different mathematical or psychoneural contexts and, optionally, translate those terms into his or her own equivalent or alternative definitions.

12. Normalization of $A$ as a fraction of the total display area may provide a more convenient measure in dimensionless units.

13. In the search arrays generated by Motter and Belky (1998b, p. 1008), the target is more likely placed in central than in peripheral regions, in inverse proportion to the distance $r$ from the screen center. Namely, the radial probability density of placing of the target between a radius $r$ and $r+d r$ is constant, rather than proportional to $r$, as in the arrays that we generated with a uniform distribution of target locations. The effect of the display boundary may thus be somewhat reduced in the Motter-Belky array structure, since that offers a greater chance of capturing the target promptly near the starting central fixation (Keech et al., 2009). Corresponding to a diminished effect of the display boundary, there could also be a somewhat diminished prevalence of eye trajectories circulating around that. Using the original data in Motter and Belky (1998a, 1998b), it may be possible to test this hypothesis quantitatively, which could increase our understanding of both the effects of the display boundary and the mechanisms of generation of circulating patterns in the eye movement trajectories. More generally, we expect, or at least we hope, that other researchers will apply or adapt our or similar techniques and measures to different experimental configurations and situations, since only that may ultimately confirm the generality of our findings and advance our understanding of the presence and origins of scanning patterns in visual search, whether circulating as a result of boundaries or otherwise.

14. The fact that eye trajectories tend to spiral outward from the display center with a forward bias also tends to progressively reduce the probability of return to central locations inspected initially. But that alone still cannot account for the great reduction in return rates that is observed with an increasing number of intervening saccades (Keech et al., 2009; Keech \& Resca, in press). 


\section{APPENDIX A}

\section{Autocorrelation Functions of Saccade Velocities and Direction Cosines}

In a stationary regime or ensemble, an autocorrelation function, such as that defined in Equation 2 of this article, has the following properties, which are critical for interpreting the behavior of a system subject to fluctuations (Pathria, 1996):

1. $K\left(t_{1}, t_{2}\right)=K(\tau)$ depends only on the time increment $\tau$.

2. $K(0)$ then represents the mean square value of the stationary variable.

3. $K(0)$ is positive and no less than any $|K(\tau)|$ for $\tau>0$.

4. $K(\tau)$ is symmetric in $\tau$; that is, $K(\tau)=K(-\tau)=K(|\tau|)$.

5. When $\tau$ becomes large as compared with a characteristic time $\tau^{*}, K(\tau)$ vanishes, meaning that $\mathbf{v}\left(t_{1}\right)$ and $\mathbf{v}\left(t_{1}+\tau\right)$ become uncorrelated, losing "memory" in between.

In our application, $\mathbf{v}(t)=\left\{v_{x}(t), v_{y}(t)\right\}$ represents the two-dimensional saccade velocity as projected on the display, and time is merely the integer counting of the saccades in each trial. Averaging over the saccade counting in each trial for a given array size, and then averaging over all such trials, the saccade velocity autocorrelation function acquires the discrete form

$$
K(l)=\frac{1}{M} \sum_{m=1}^{M} \frac{1}{N_{m}-l} \sum_{k=1}^{N_{m}-l} \mathbf{v}(m, k) \cdot \mathbf{v}(m, k+l) .
$$

In Equation A1, $N_{m}$ represents the number of saccades in the $m$ th trial, $M$ represents the total number of recorded trials for a given array size, and the sum over $k$ is replaced by zero if $N_{m} \leq l$. Since there were 90 target-present trials for 7 participants (in each age group), $M$ could have always taken the maximum value of 630 . However, whenever a participant did not press either button until after the 4-sec time limit had expired for a given trial, the following trial was aborted as soon as a button was pressed. Although that never occurred for the 12- and 24-distractor arrays, there was a reduction to 621 and to 605 in the number $M$ of recorded trials for the older adults searching the 48-distractor and 96-distractor arrays, for example. On average, the older adults made 3.3, 4.1, 6.5, and 8.4 saccades in the 12-, 24-, 48-, and 96-distractor arrays, respectively. Thus, for the older adults, there were approximately $2,079,2,583,4,037$, and 5,082 terms contributing to $K(l)$ averages in Equation A1 for the corresponding array sizes.

From those data, we also estimated as follows standard errors for saccade direction cosine autocorrelations $R(l)$, defined in Equations 1 and 3. Since $0.5^{\circ}$ represents the spatial resolution of the eyetracker and $6.5^{\circ}$ is an average saccade amplitude, the angular resolution in an average saccade direction is given approximately by the ratio $\delta \theta \approx 0.5 / 6.5 \approx 0.077$ radians. Thus, the average cosine resolution is about $\cos (\pi / 2-\delta \theta) \approx \delta \theta \approx 0.077$ or better. By dividing that 0.077 by the square root of the number of terms that contribute to $R(l)$ on average, we estimated standard errors for $R(l)$, which are shown as error bars in Figures 1A-1D and correspond to \pm 0.0017 , $\pm 0.0015, \pm 0.0012$, and \pm 0.0011 relative values for 12 -, 24 -, 48 -, and 96 -distractor arrays, respectively.

Note that those standard error estimates for $R(l)$ derive from the instrumental spatial resolution, independently of any variability among human participants. In those estimates, as in Equation A1 for that matter, we thus regarded all trials as if they had been globally performed by just one aggregate subject, rather than by 7 actual participants (in each age group). Sampling of saccades by different participants may introduce another kind of sampling error. However, it has been noted (Loftus \& Masson, 1994) that "given the irrelevance of inter-subject variance in a within-subjects design, it can legitimately be ignored for purposes of statistical analysis" (p. 481). Furthermore, the within-subjects contribution to error is typically small (Loftus \& Masson, 1994; Masson \& Loftus, 2003). We certainly expected that in our case, since each participant still contributed a large number (hundreds) of saccades to $R(l)$ for each $l$ value. We did not compute that error, however. If we had, we could have more precisely determined further small confidence interval contributions to $R(l)$ errors, as suggested by Loftus and Masson.

We should also point out that in this analysis, we have considered only trials on which the target was present in order to consistently compare the corresponding $R(l)$ with those obtained later in computer simulations (Keech, 2006; Keech \& Resca, in press). However, autocorrelations including all trials $(M \approx 693)$ do not differ appreciably from those presented in Figures $1 \mathrm{~A}-1 \mathrm{D}$, which include only target-present trials $(M \approx 630)$ but have already essentially converged.

Finally, recall that we have considered the saccade direction cosine autocorrelation $R(l)$ essentially for the purpose of comparing it with that of directional randomness between different saccades. Another possibility for performing that comparison computationally would be to randomly reshuffle the order of the saccades in each trial and then recompute the corresponding baseline autocorrelation $B(l)$. Subtracting that from the actual $R(l)$ should yield its beyond-chance component. In our application, however, there are too few saccades in too many trials to ensure an effective reshuffling randomization, thus allowing some spurious correlation to remain in $B(l)$. So, we prefer to assume that $B(l)=\delta_{l, 0}$ ideally represents the cosine autocorrelation of an unconstrained random walk, and we compare the actual $R(l)$ directly with that. Various comparisons with computer simulations of random-walk trajectories with saccade characteristics and with or without boundary constraints are shown and discussed in Appendix B. 


\section{APPENDIXA (Continued)}

\section{Fourier Transforms of Autocorrelation Functions and Power Spectra}

The Fourier transform of the autocorrelation function $K(\tau)$ of a stationary variable generates its spectral density $S(f)$, and conversely, as

$$
S(f)=\int_{-\infty}^{\infty} K(\tau) e^{-i(2 \pi f \cdot \tau)} d \tau
$$

and

$$
K(\tau)=\int_{-\infty}^{\infty} S(f) e^{i(2 \pi \tau \cdot f)} d f
$$

Because of Properties 1-4 of $K(\tau), S(f)$ is also a symmetric and real function of the frequency $f$, which makes it more convenient to define the power spectrum density as

$$
w(f)=2 S(f), \text { for } f \geq 0 \text { only. }
$$

These results form part of what is generally known as the Wiener-Khinchin theorem (Pathria, 1996).

We are actually interested in the saccade direction cosine autocorrelation $R(l)$, which has a discrete form. Its power spectrum is then obtained as a discrete Fourier transform (DFT),

$$
W(k)=\frac{4}{2 L-1}\left\{\frac{1}{2} R(0)+\sum_{l=1}^{L-1} R(l) \cos \left(2 \pi l \frac{k}{2 L-1}\right)\right\} .
$$

This assumes that $R(l)=R(-l)$ has real values assigned symmetrically for $l=-L+1, \ldots,-1,0,1, \ldots, L-1$, and that $R(l)$ vanishes for $|l| \geq L$. Then $R(l)$ is periodically extended to all $l$ integers, formally, with a period of $N=2 L-1$, which in turn requires that $R(L-1)=0$ also. Correspondingly, $W(k)=W(-k)$ has the same periodicity, with an inverse DFT,

$$
R(l)=\frac{1}{2} W(0)+\sum_{k=1}^{L-1} W(k) \cos \left(2 \pi l \frac{k}{2 L-1}\right) .
$$

We may further regard $f_{k}=k /(2 L-1)$, with $k=0,1, \ldots, L-1$, as the $L$ frequencies of the discrete power spectrum $W\left(f_{k}\right) \equiv W(k)$ of $R(l)$. In our computations, we assume that $L=16$ (cf. Figures 1 and 2 ). For white noise, $R(l)=R(0) \delta_{l, 0}$, we obtain a constant

$$
W(k)=\frac{4}{2 L-1}\left\{\frac{1}{2} R(0)\right\}
$$

for all the $L$ frequencies $f_{k}$ or $k$ values. Note that, in all cases,

$$
R(0)=\frac{1}{2} W(0)+\sum_{k=1}^{L-1} W(k)=1
$$

provides the normalization consistent with our definition of the discrete power spectrum.

In our application, the lag $l$ represents directly the number of intervening saccades in the autocorrelation function $R(l)$ defined in Equation 3. Mathematically, we regard $l$ as the integral independent variable, ranging from zero to $L-1$, in the direct space on which the function $R(l)$ is defined as the dependent variable. Using Equation A4, we then generate the discrete Fourier cosine transform $W(k)$ of $R(l)$. That $W(k)$ is a function that acts as the dependent variable on the reciprocal space of the integral independent variable $k$, also ranging from zero to $L-1$. Of course, $l$ and $k$ vary independently of each other in different spaces, albeit of equal dimension. For notational convenience, by merely rescaling $f_{k}=k /(2 L-1)$, we have also introduced a frequency space completely equivalent to reciprocal space.

Now, Equations A4 and A5 represent integral transforms of each other, with the function $\cos \left(2 \pi l \cdot f_{k}\right)$ acting as kernel in the corresponding summations over $l$ and $f_{k}$, respectively (in Equation A2, integrals replace those summations, more generally). When the product $l \cdot f_{k}$ exceeds one, resulting from the increase in the independent variable over which the summation is carried, the $\cos \left(2 \pi l \cdot f_{k}\right)$ function oscillates more and more rapidly. Such rapid oscillations tend to average out and progressively reduce the contributions that either $R(l)$ or $W\left(f_{k}\right)$ makes to the other, through the summations in Equations A4 and A5, respectively. As a result, a characteristic behavior of Fourier transforms emerges - namely, that components $W\left(f_{k}\right)$ of the power spectrum at higher and higher frequencies $f_{k}$ are mostly affected by components of the autocorrelation $R(l)$ at shorter and shorter range $l$, since only those $l$ components are spared from destructive interference of the cosine kernel in the summation in Equation A4. Conversely, components of the autocorrelation $R(l)$ at longer and longer range $l$ are mostly affected by components $W\left(f_{k}\right)$ of the power spectrum at lower and lower frequencies $f_{k}$, contributing predominantly to the summation in Equation A5. Such typical behavior, however, is not readily formulated as a rigorous mathematical theorem, because it does not depend exclusively on the general oscillatory property of the Fourier transform kernel but, also, on the specific form that the function that is integrated by that kernel may have in any particular case. Thus, in our application, we simply use this general property as a rule of thumb, according to which relatively large components $W\left(f_{k}\right)$ of the power spectrum at high frequencies $f_{k}$ correspond to appreciable components of the autocorrelation $R(l)$ at short range $l$, and conversely at 


\section{APPENDIX A (Continued)}

low frequencies. Since $2 \pi$ represents the basic period of oscillation of $\cos \left(2 \pi l \cdot f_{k}\right)$ as a function of its whole argument, we can then assume an approximate relation $l \sim 1 / f_{k}$ for finding which appreciable $R(l)$ components may derive from $W\left(f_{k}\right)$ dominant components or vice versa. Having set $f_{k}=k /(2 L-1)$ and $L=16$ in our application, we have correspondingly $l \sim 31 / k$.

Whereas the lag $l$ has a direct and immediately intuitive interpretation-namely, that of the number of intervening saccades in the autocorrelation function $R(l)$ defined in Equation 3-what is the interpretation of the frequency $f_{k}$ in $W\left(f_{k}\right)$ ? This is not as immediately intuitive, other than generally thinking of a frequency as the inverse of the period of any sinusoidal oscillation, meaning how frequently that oscillation recurs. Thus, a short lag $l$ in $R(l)$ suggests a mapping to a high frequency $f_{k}$ in $W\left(f_{k}\right)$, and conversely, once again. Although an intuitive interpretation of frequencies is definitely more immediate in some applications than in other ones, practitioners of harmonic analysis always develop and use as a guide at least a "mathematical" intuition of frequency space, spectral functions, and the like. Our application demonstrates some of the benefits that derive from using a spectral analysis, despite the increased demand and reliance on its mathematical formulation and corresponding intuition.

APPENDIX B

Circulation and Area Measures for Perfectly Circular Trajectories and Random Walks

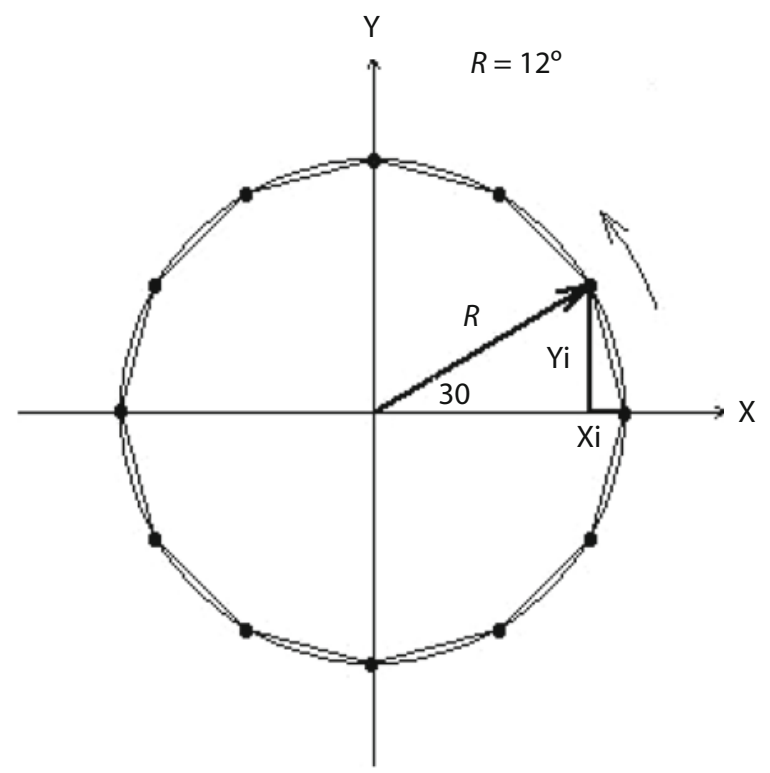

Figure B1. Perfectly circular/polygonal search trajectory.

Here, we investigate distributions and properties of the circulation $C$, area measure $A$, and imbalance $I$ for opposite limits of perfectly circular trajectories and random walks, plus their autocorrelations and power spectra.

Figure B1 shows a circle of radius $R=12^{\circ}$ and an idealized polygonal trajectory of 12 equal-amplitude saccades inscribed in it. The initial saccade along the search trajectory reaches a point along the circle at an angle of $30^{\circ}$, or $\pi / 6$ radians, above the horizontal. The saccade amplitude is about $R \cdot \pi / 6$, or about $6^{\circ}$, while the horizontal displacement is $\Delta X=X_{i=1}=$ $R \cdot[1-\cos (\pi / 6)]$ and the vertical displacement is $\Delta Y=Y_{i=1}=$ $R \cdot \sin (\pi / 6)$. At this point, the circulation $C$ is still zero, since no angle shift has yet occurred. In the next step, the total horizontal displacement becomes $\Delta X=X_{i=2}=R \cdot[1-\cos (\pi / 3)]$, and the total vertical displacement becomes $\Delta Y=Y_{i=2}=R \cdot \sin (\pi / 3)$. Thus, by definition, $A=R^{2} \cdot \sin (\pi / 3) \cdot[1-\cos (\pi / 3)]$ and $C=1$ in magnitude (or for a positive counterclockwise convention). Figure B2 shows the behavior of $\Delta X$ and $\Delta Y$ as the counterclockwise search progresses. Clearly, $\Delta X$ reaches its maximum value of $2 R$ after the 6th saccade, when $C=5$, whereas $\Delta Y$ is maximized at $2 R$ after the 9th saccade, when $C=8$. Figure B3 shows the corresponding dependence of $A$ on $C$.

Given the definition of A - that is, $A=\Delta X \cdot \Delta Y$ - the increase of $A$ with $C$ is evidently nonlinear. Furthermore, $A$ reaches its maximum value for $C=8$, corresponding to a turning around of $270^{\circ}$. As the trajectory continues, $A$ stays at that maximum value,

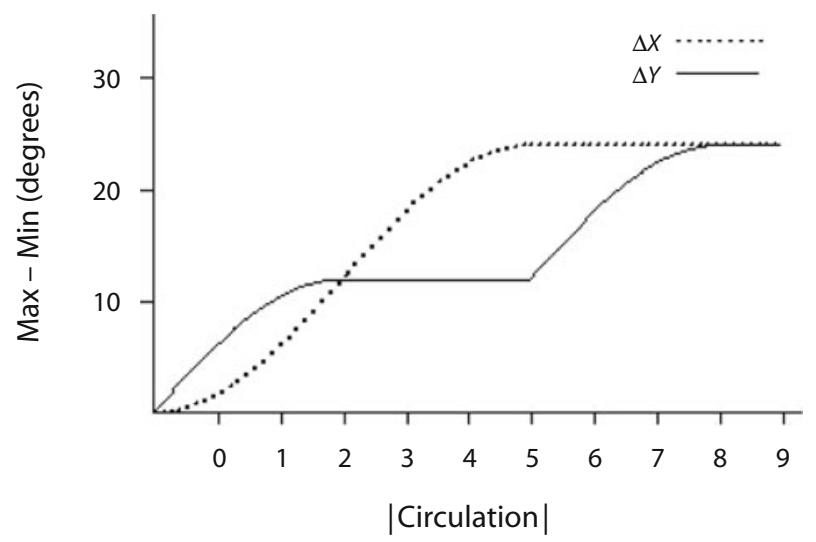

Figure B2. Increase of $\Delta X$ and $\Delta Y$ with increasing $|C|$ for the perfectly circular/polygonal search trajectory in Figure B1.

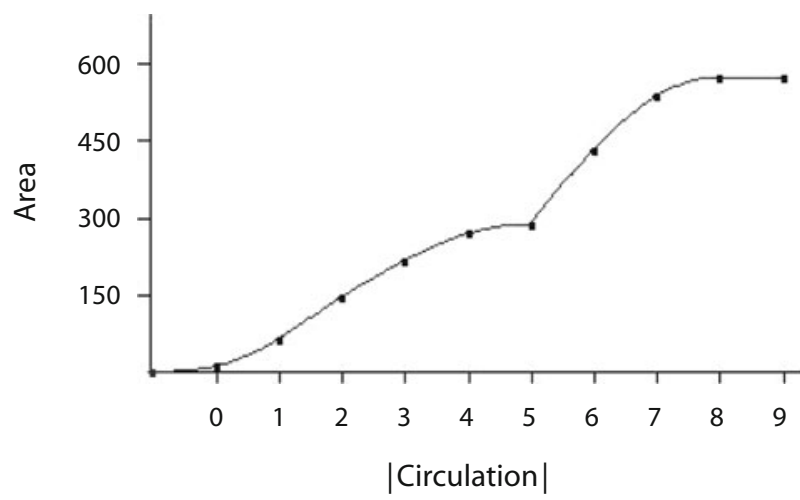

Figure B3. Area measure $A$ as a function of the circulation magnitude $|C|$ for the perfectly circular/polygonal search trajectory shown in Figure B1. 


\section{APPENDIX B (Continued)}

while $C$ still increases up to its maximum value of 11 , where the trajectory closes on itself. So, $A$ saturates at $C=8$. In particular, $A$ increases initially in proportion to the cube of $C$ for a perfectly circular trajectory, since at small angles,

$$
\sin (\theta) \cdot[1-\cos (\theta)] \approx \frac{\theta^{3}}{2} .
$$

With regard to the imbalance, any progression along a circular or convex trajectory keeps it at $100 \%$, by definition.

The opposite of perfectly organized circular motion is random walk, which cannot be determined analytically but for which we can perform computer simulations. Our main purpose is actually that of generating a baseline comparison for the observed eye trajectories, in which the psychoneural mechanisms underlying the visual search are virtually removed, whereas the geometric-spatial constraints on the search are retained. Namely, we maintain a rectangular boundary of $24.4^{\circ} \times 16.3^{\circ}$, corresponding to the experimental display size, and we require the search to start at the center of the display. Then we generate 60,000 simulated trials with saccades of identical amplitude, which may be taken as $6.5^{\circ}$, for example, corresponding to the average for 96-distractor arrays searched by the older adults (cf. Figure 7D in Keech et al., 2009). The large number of simulated trials $(60,000)$ practically eliminates standard errors for our baseline comparison. On the other hand, we wish to preserve for that comparison the saccade number distribution that is experimentally observed: For visual searches of 96-distractor arrays by the older adults, that would be the thin line shown in Figure 2D in Keech et al. (2009). Taking the corresponding percentages for 60,000 trials, we generate the correct number of saccades for each simulated trial. Figure B4 shows an example of such a random-walk trajectory.

Figure B5 shows (A) the discretized distribution of the circulation $C$ and (B) a scatterplot of the area measure $A$ and the circulation $C$, for 60,000 random-walk simulations corresponding to 96-distractor arrays searched by the older adults. Figure B5 thus provides the baseline comparison for Figure 6 of the actual search, having virtually removed the effects of the psychoneural mechanisms, but not those of the geometric-spatial constraints on the visual search. In contrast to the actual search, there is no

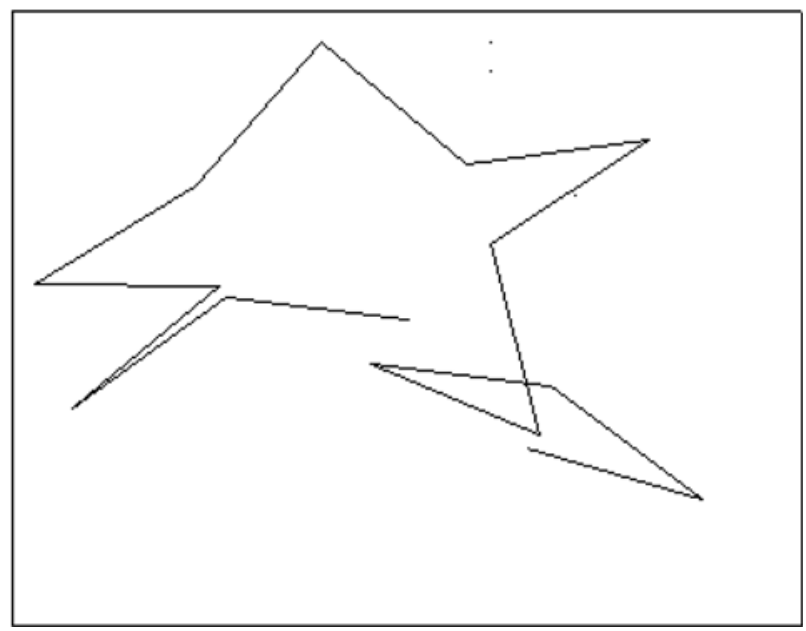

Figure B4. Random-walk trajectory with a rectangular boundary constraint. The trajectory begins at the center and consists of 14 constant-amplitude saccades. The corresponding measures of circulation and imbalance are $C=3$ and $I=8 / 13$.

\section{A Circulation Distribution}

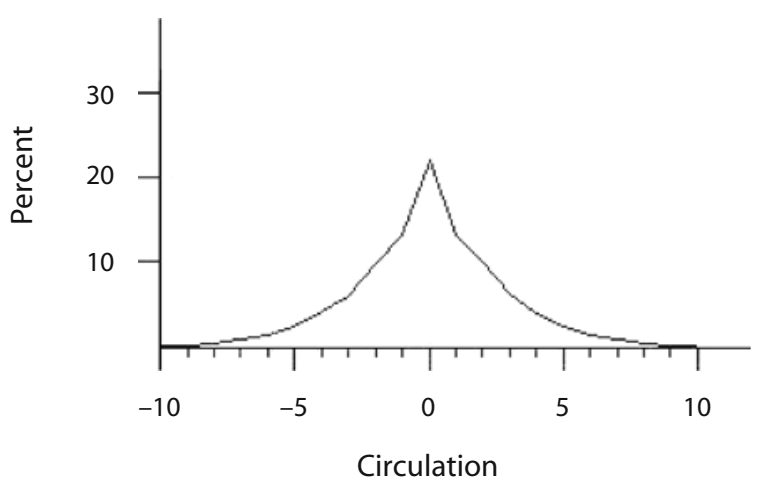

B

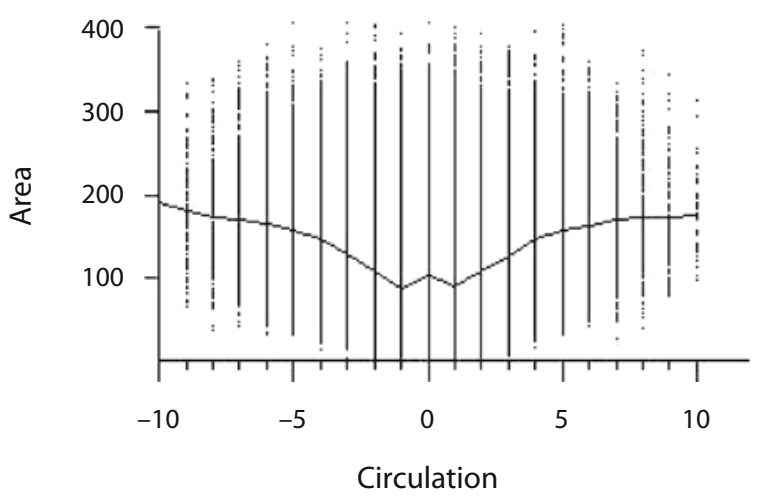

Figure B5. Circulation $C$ distribution (A) and area-circulation scatterplot (B) for 60,000 random-walk simulations with a rectangular boundary constraint, corresponding to 96-distractor arrays searched by the older adults. 


\section{APPENDIX B (Continued)}

bimodality in the $C$ distribution for the random-walk trajectories. Namely, the most probable value of $C$ is strongly peaked at $C=0$ in Figure B5A, indicating no prevalence of circulating trajectories. Correspondingly, the imbalance distribution for 60,000 random-walk simulations with a rectangular boundary constraint shows a prevalence of the no-imbalance peak at $I=0.5$. Similar results and conclusions are obtained with other random-walk trajectories, simulating other array sizes with boundaries, searched by either young or older adults.

Figure B6A shows the saccade direction cosine autocorrelation for the same 60,000 random-walk simulated trajectories. Evidently, the rectangular boundary constraint causes negative $R(l)$ for all $l>0$, resulting from the prevalence of direction reversals. That clearly differs from the corresponding Figure 1D

A

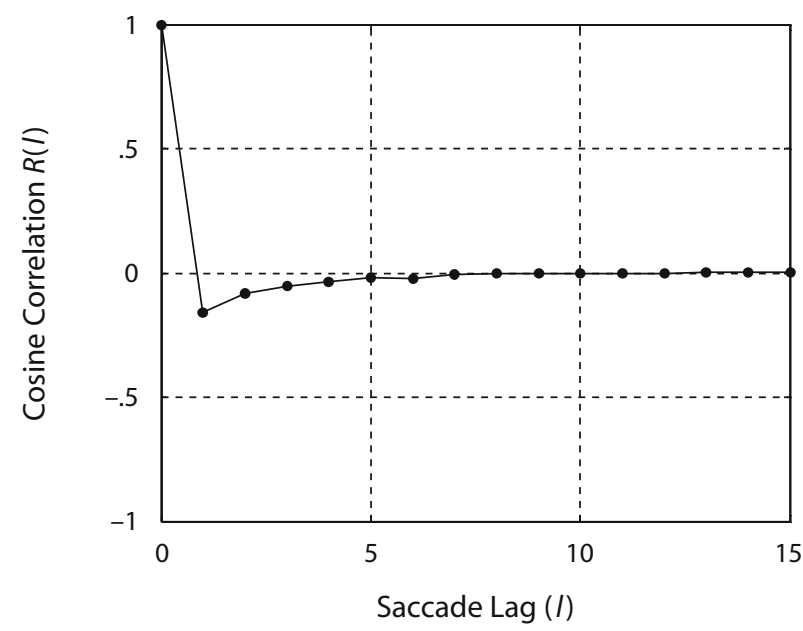

B

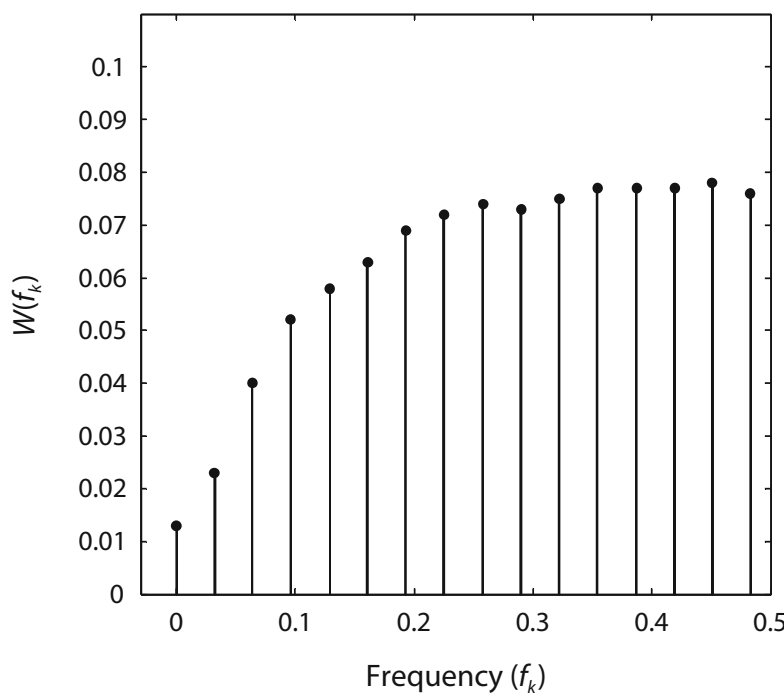

Figure B6. Saccade direction cosine autocorrelation (A) and its power spectrum $(B)$ for 60,000 random-walk simulations with a rectangular boundary constraint, corresponding to those of visual searches of 96-distractor arrays by the older adults.

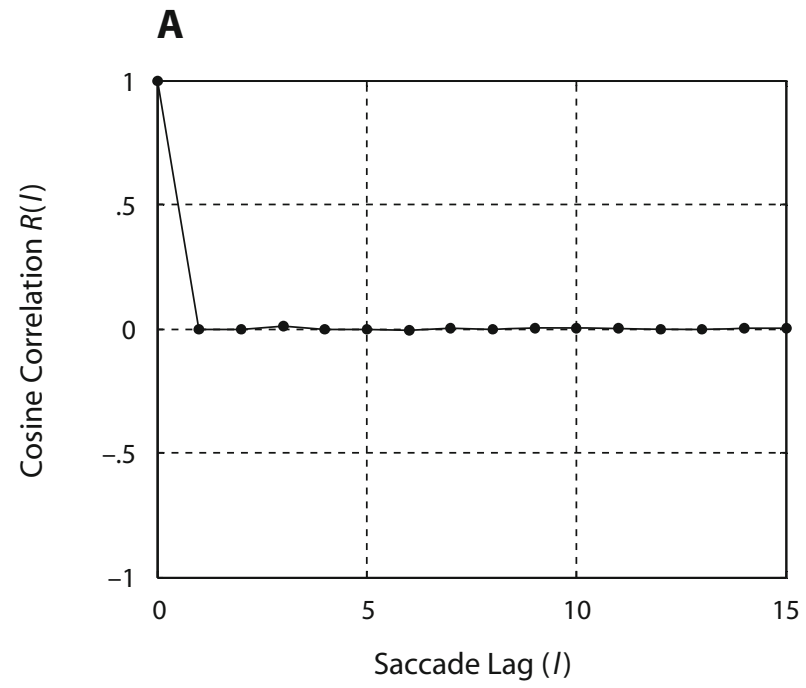

B

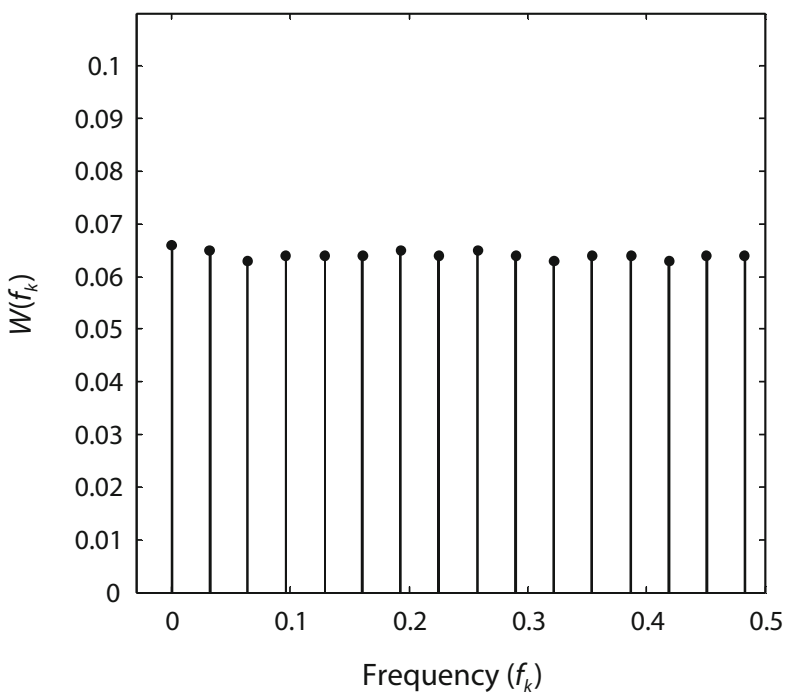

Figure B7. Saccade direction cosine autocorrelation (A) and its power spectrum (B) for 60,000 random-walk simulations without any boundary constraint, corresponding to those of visual searches of 96-distractor arrays by the older adults.

for the human search, in which $R(l=1)$ is positive, as a result of the prevailing forward bias, which is present in the human (but not in the simulated) searches. Figure B6B shows the power spectrum for the 60,000 random-walk trajectories, corresponding to Figure 2B for 96-distractor arrays searched by the older adults. Evidently, these two power spectra differ substantially, although the retention of the rectangular boundary constraint in the simulations still causes the power spectrum in Figure B6B to depart considerably from that of a constant white noise.

As a final comparison, even the geometric-spatial constraints can be removed in the computer simulations, thus allowing completely random walks. Figure B7 shows the saccade direction cosine autocorrelation (A) and its corresponding power spectrum (B) for 60,000 unbounded random-walk simulations, cor- 


\section{APPENDIX B (Continued)}
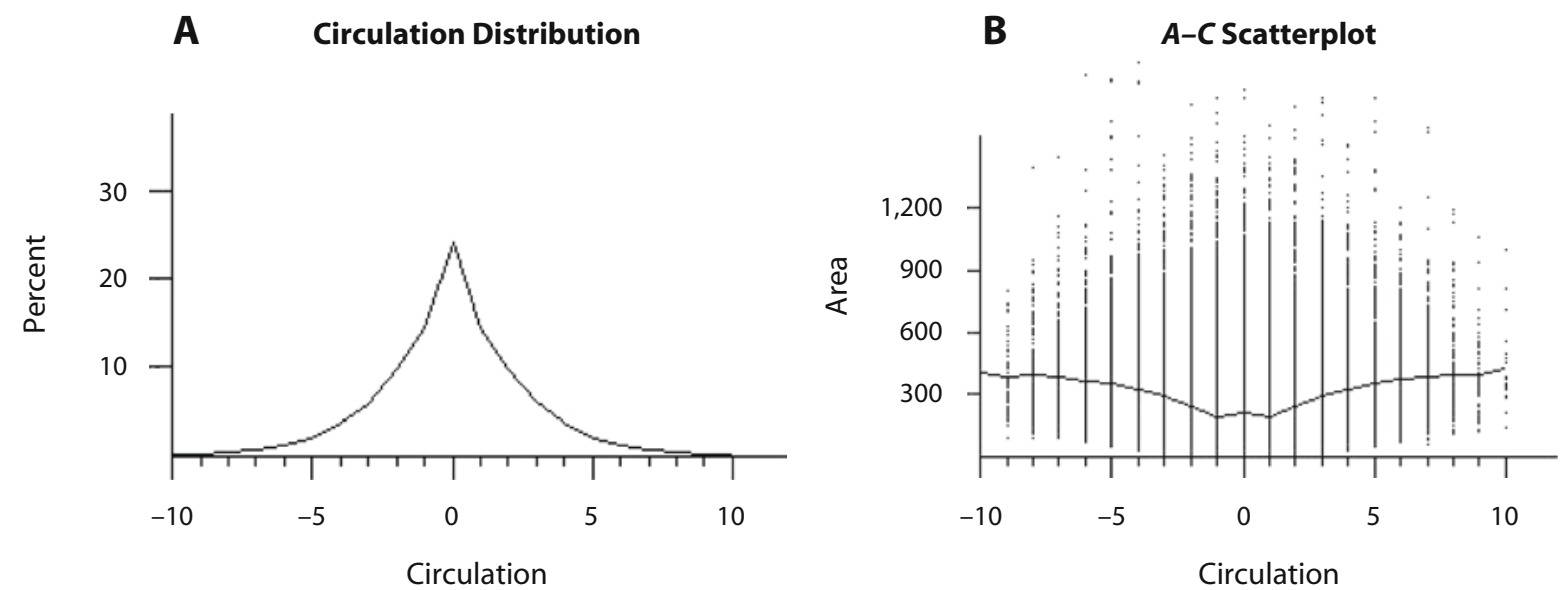

Figure B8. Circulation $C$ distribution (A) and area-circulation scatterplot (B) for 60,000 random-walk simulations without any boundary constraint, corresponding to 96-distractor arrays searched by the older adults.

responding to trials for 96-distractor arrays searched by the older adults. Now the saccade direction cosine autocorrelation virtually matches the expected baseline autocorrelation $B(l)=\delta_{l, 0}$, and the corresponding power spectrum is virtually flat, as would be expected for white noise. The distribution and scatterplot for $C$ and $A$, shown in Figure B8, remain qualitatively similar to those shown in Figure B5, since the prevalence of spiraling or circulating trajectories was already eliminated therein, even in the presence of the geometric-spatial constraints. Note, however, that much wider area measures are typically subtended by random walks without boundaries, naturally. 